\title{
Interferometric Monitoring of Gamma-ray Bright AGNs: Measuring the Magnetic Field Strength of 4C +29.45
}

\author{
S. Kang ${ }^{1,2}$ S.-S. Lee ${ }^{1,2}$ J. Hodgson ${ }^{1,3}$ J.-C. Algaba ${ }^{4}$ J. W. Lee ${ }^{1}$ J.-Y. Kim ${ }^{5}$ J. Park ${ }^{6}$ M. Kino ${ }^{7,8}$ D. Kim ${ }^{9}$ and S. Trippe ${ }^{9}$
}

1 Korea Astronomy and Space Science Institute, 776 Daedeok-daero, Yuseong-gu, Daejeon 34055, Korea e-mail: sslee@kasi.re.kr

2 University of Science and Technology, Korea, 217 Gajeong-ro, Yuseong-gu, Daejeon 34113, Korea

3 Department of Physics and Astronomy, Sejong University, 209 Neungdong-ro, Gwangjin-gu, Seoul, South Korea

4 Department of Physics, Faculty of Science, University of Malaya, 50603 Kuala Lumpur, Malaysia

5 Max-Planck-Institut für Radioastronomie, Auf dem Hügel 69, D-53121 Bonn, Germany

${ }^{6}$ Institute of Astronomy and Astrophysics, Academia Sinica, 11F of Astronomy-Mathematics Building, AS/NTU No.1, Sec.4, Roosevelt Rd, Taipei 10617, Taiwan, R.O.C.

7 National Astronomical Observatory of Japan, 2-21-1 Osawa, Mitaka, Tokyo 181-8588, Japan

8 Kogakuin University of Technology \& Engineering, Academic Support Center, 2665-1 Nakano, Hachioji, Tokyo 192-0015, Japan

9 Department of Physics and Astronomy, Seoul National University, Gwanak-gu, Seoul 08826, Korea

\begin{abstract}
Aims. We present the results of multi-epoch, multi-frequency monitoring of a blazar $4 \mathrm{C}+29.45$, which was regularly monitored as part of the Interferometric Monitoring of GAmma-ray Bright Active Galactic Nuclei (iMOGABA) program - a key science program of the Korean Very long baseline interferometry Network (KVN).

Methods. Observations were conducted simultaneously at 22, 43, 86, and $129 \mathrm{GHz}$ during the 4 years from 5 December 2012 to 28 December 2016. We also used additional data from the $15 \mathrm{GHz}$ Owens Valley Radio Observatory (OVRO) monitoring program.

Results. From the $15 \mathrm{GHz}$ light curve, we estimated the variability time scales of the source during several radio flux enhancements. We found that the source experienced 6 radio flux enhancements with variability time scales of 9-187 days during the observing period, yielding corresponding variability Doppler factors of 9-27. From the multi-frequency simultaneous KVN observations, we were able to obtain accurate radio spectra of the source and hence to more precisely measure the turnover frequencies $v_{\mathrm{r}}$ of synchrotron self-absorbed (SSA) emission with a mean value of $\overline{v_{\mathrm{r}}}=28.9 \mathrm{GHz}$. Using jet geometry assumptions, we estimated the size of the emitting region at the turnover frequency. We found that the equipartition magnetic field strength is up to two orders of magnitudes higher than the SSA magnetic field strength $(0.001-0.1 \mathrm{G})$. This is consistent with the source being particle dominated. We performed a careful analysis of the systematic errors related to making these estimations.

Conclusions. From the results, we concluded that the equipartition region locates upstream the SSA region.
\end{abstract}

Key words. galaxies: active — galaxies: jets — BL Lacertae Objects: individual $(4 \mathrm{C}+29.45)$

\section{Introduction}

Blazars are a category of Active Galactic Nuclei (AGNs) where the relativistic jet is oriented almost directly toward us (Angel \& Stockman 1980). Because of the small viewing angle (e.g., $\leq 5$ degrees, Jorstad et al. 2017), the emission from most of the blazars shows dynamic variations of total flux density and polarization, and many of these blazars are highly Doppler boosted (Madau et al. 2013). Very long baseline interferometry (VLBI) studies of relativistic jets have revealed that the jets are highly collimated and exhibit acceleration (Jorstad et al. 2017; Homan et al. 2015). Many studies have tried to explain how the jets can be accelerated and collimated (e.g., Homan et al. 2015; Nakamura \& Asada. 2013). One potential scenario is that the magnetic fields around the jet accelerate and collimate the jet (Begelman, Blandford \& Rees 1984; Komissarov et al. 2007). Meanwhile, shocks in jets make the magnetic fields aligned or tangled (Marscher et al.|2008). Therefore, it is important to study the magnetic field properties around blazar jets. As a well-known example for such studies, M87, one of the brightest and closest AGNs, has been densely studied, including extensive investigation of its magnetic field properties (Asada \& Nakamura 2012;
Asada et al.2014; Hada et al. 2016; Kim et al.|2018b; Mertens et al.2016, Nakamura et al. 2018, EHTC 2019, Park et al.|2019a c).

One way to study magnetic fields is to measure the polarization of the emission. When relativistic electrons pass through magnetic fields, these electrons emit synchrotron radiations which can be linearly polarized by up to $70 \%$ (Hales et al. 2017). Many studies have performed VLBI polarization observations of the jets in order to reveal the structure of the magnetic fields. (e.g., Gabuzda 2017). Also, Agudo et al.(2018) found that the magnetic fields measured at shorter wavelengths were more ordered than at longer wavelengths by comparing the fractional polarization at 86 and $230 \mathrm{GHz}$. However, it is rare to report the results of directly measuring the magnetic field strength from polarimetric measurements.

Another way of studying magnetic fields is to measure the apparent core shifts of optically thick synchrotron selfabsorption (SSA) emission at different frequencies (Lobanov 1998, Pushkarev et al. 2012, Lee et al. 2016a). Lobanov (1998) estimated the magnetic field strengths of several radio sources using the core shift. In their estimation, they assumed that the region at $1 \mathrm{pc}$ from the central engine is under equipartition condition. Equipartition means that magnetic field and relativistic 
particle energies are equal. Under these assumptions, they derived the magnetic field strength $B_{\text {core }}$ of various relativistic jets measuring strengths ranging from $0.3 \mathrm{G}$ to $1.0 \mathrm{G}$. This method has been used by various other authors finding broadly consistent results (e.g., Algaba et al. 2012; Kovalev. 2008; Pushkarev et al. 2012).

A way to directly measure the magnetic field strengths is to measure the turnover frequency of SSA emission, the size of the emission at the turnover frequency, and the flux density at the turnover frequency (Marscher 1983). This method has been applied by several authors including Lee et al. (2017) that estimated the magnetic field strength in the relativistic jet from a bright blazar S5 $0716+714$ to be $\leq 5 \mathrm{mG}$. Similarly, Algaba et al. (2018b) derived a magnetic field strength of $0.1 \mathrm{mG}$ in the flat spectrum radio quasar $1633+382(4 \mathrm{C} 38.41)$ and Lee et al. (2020) obtained a magnetic field strength of $0.95-1.93 \mathrm{mG}$ of magnetic field strength for a blazar OJ287.

$4 \mathrm{C}+29.45$ (also known as $1156+295$, J1159+2914, and TON 599) is classified as a blazar (Veron-Cetty \& Veron 2010) with a redshift of $\mathrm{z}=0.729$ (Zhao et al.2011; Wang et al. 2014). It shows a wide time scale range of variations from hours to years (Liu et al. 2013, Wang et al. 2014, Aharonian et al. 2007; Raiteri et al. 2013). Many previous studies have attempted to explain these variations (Liu et al. 2013; Wang et al. 2014, Aharonian et al. 2007, Raiteri et al. 2013). Liu et al. (2013) explained the cm-wave intra-day variations as being due to interstellar scintillation (ISS). Wang et al. (2014) interpreted the long-term periodic variations from 1.7 to $7.5 \mathrm{yr}$ as being due to pressure driven oscillations from the accretion disk. Moreover, the source shows strong activity in $\gamma$-rays. The Fermi-Large Area Telescope (LAT) detected high $\gamma$-ray activity of the source on 20 November 2015 (MJD 57346) (Prince 2019), with a daily averaged $\gamma$-ray flux of $4 \times 10^{-7}$ photons $\mathrm{cm}^{-2} \mathrm{~s}^{-1}$. A possible correlation between $\gamma$-ray flaring and radio variability has not been reported and will be studied in a follow-up paper.

In this paper, we aim to study the magnetic field strength of $4 \mathrm{C}+29.45$ using Korean VLBI Network (KVN) multi-frequency simultaneous observations and combining with other publicly available data. We introduce our observations and data reduction scheme in Section 2, and show our results and our data analysis in Section 3 . Then, we discuss our results in Section 4 and finally draw our conclusions in Section 5

\section{Observations and Data Reduction}

\section{1. iMOGABA observations}

$4 \mathrm{C}+29.45$ is a target as part of a monitoring program using the KVN. This program is known as Interferometric Monitoring of GAmma-ray Bright AGNs (iMOGABA) and has, since 5 December 2012 (MJD 56266), been conducting almost monthly observations of over 30 sources at 22, 43, 86, and $129 \mathrm{GHz}$ simultaneously (Lee et al. 2016b). The purpose of the iMOGABA program is to study the correlation between radio and $\gamma$-ray light curves in $\gamma$-ray bright AGNs and to constrain the characteristics of the $\gamma$-ray flares originating in AGNs (Algaba et al. 2018ab; Hodgson et al.|2018, Kim et al.|2017,,2018, Lee et al.|2017;:Park et al. 2019b). The data presented in this paper are of the source $4 \mathrm{C}+29.45$ and were conducted for about 4 years from 5 December 2012 (MJD 56266) to December 2016 (MJD 57750). This yielded 33 epochs, which were observed in snapshot modes consisting of 2 to 7 scans with 5-min duration at intervals of several hours. There is a mean cadence of $\sim 30$ days with some gaps in time due to summer maintenance. We set the starting frequencies at $21.7,43.4,86.8$ and $129.3 \mathrm{GHz}$ with a bandwidth of $64 \mathrm{MHz}$, as an integer frequency ratio, allowing for the frequency phase transfer technique to be applied allowing for increased detection sensitivity at higher frequencies (Algaba et al. 2015; Hodgson et al.2016). Antenna pointing offsets were checked at each station for every scan by conducting cross-scan observations for each target source. Atmospheric opacities were measured every hour by conducting sky tipping measurements. Further detailed observation schemes are described in Lee et al. (2016b).

\subsection{Data reduction and imaging}

Observational data were recorded using the Mark 5B system with a recording rate of $1 \mathrm{Gbps}$. Recorded data at the three antennas were correlated with an accumulation period of 1 second, using the DiFX software correlator at the Korea-Japan Correlation Center in Daejeon, South Korea. Correlated data were reduced using the Astronomical Image Processing System (AIPS) as part of the KVN pipeline (Hodgson et al. 2016). The data reduction procedure of the KVN pipeline consists of amplitude calibration (APCAL), correction of auto-correlations (ACCOR), bandpass calibration (BPASS), fringe fitting (FRING), alignment of IF (sub-bands corresponding to the intermediate frequencies at the mixing step) phases using the IF-aligner (Marti-Vidal et al. 2012) and frequency phase transfer (FPT) (Zhao et al.2015). The main features of the KVN pipeline that are different from other pipelines are the use of the IF-Aligner and FPT. The IF-Aligner is used for calibrating delays in subbands and FPT is used for calibrating atmospheric errors at higher frequencies with lower frequency data. More details about the pipeline procedure can be found in Hodgson et al. (2016). Upper limits of the uncertainties of amplitude calibration were 5\% at 22 and $43 \mathrm{GHz}, 10 \%-30 \%$ at 86 and $129 \mathrm{GHz}$.

The reduced data were used for producing milliarcsecondscale images of the source using DIFMAP. At the beginning of the imaging procedure, we averaged the data every 30,30 , 20 , and 10 seconds at 22, 43, 86, and $129 \mathrm{GHz}$, respectively. We began by flagging outliers in amplitude. We then applied the CLEAN procedure with 10-100 iterations at loop gains of 0.05-0.1. We then applied phase self-calibration but not amplitude self-calibration due to only having three antennas (Lee et al. 2014). The CLEAN and self-calibration procedures were conducted until the residual noise is dominated by thermal random noise. As an example, CLEANed images at 22, 43, 86, and $129 \mathrm{GHz}$ obtained on 1 March 2016 are shown in Fig. 1. After the imaging procedure, we obtained beam parameters $\left(B_{\text {maj }}\right.$, $B_{\text {min }}$ and $\left.B_{\mathrm{pa}}\right)$, total CLEANed flux densities $\left(S_{\mathrm{KVN}}\right)$, peak flux densities $\left(S_{\mathrm{p}}\right)$, rms noises $\left(\sigma_{\mathrm{rms}}\right)$ and dynamic ranges $(D)$. Also, we evaluated image qualities in order to verify whether the obtained images were well modeled or not, using the ratio between the maximum flux density and the expected flux density of the residual image, $\xi_{r}=s_{r} / s_{r \text {,exp }}$ (e.g., Lee et al. 2016b). The obtained parameters are listed in Table A.1

\subsection{Gaussian model fitting}

We fitted the source images with two-dimensional circular Gaussian models. We conducted this using the MODELFIT procedure in DIFMAP until the reduced chi-squares (i.e., a fitting reliability) did not improve significantly compared with the results of the previous fitting iterations and the residual noise became comparable to a uniform distribution similar to the CLEANing procedures. After model fitting, we obtained the best fit model 

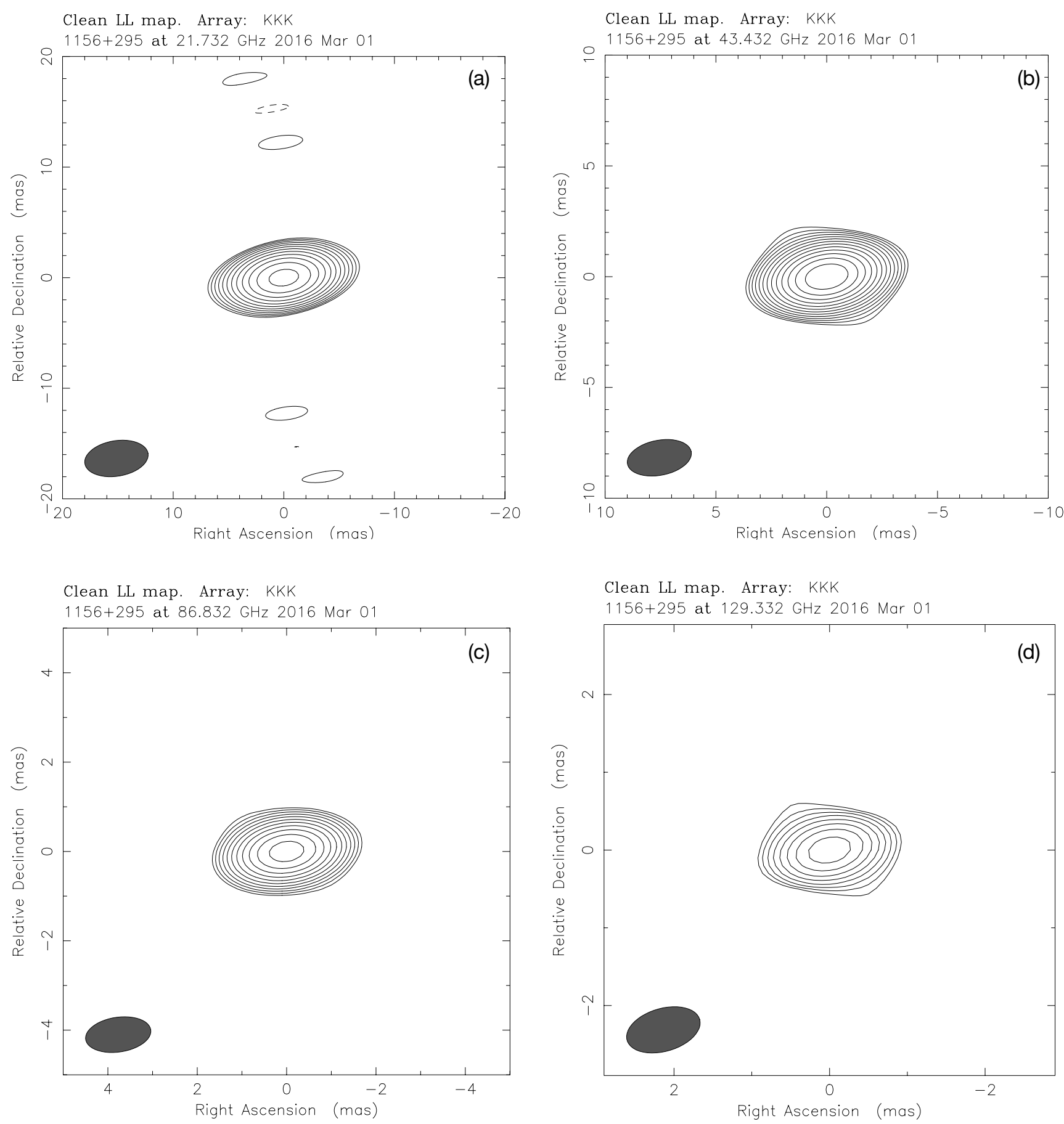

Fig. 1 Example CLEANed images of 4C +29.45 at $22 \mathrm{GHz}$ (a), $43 \mathrm{GHz}$ (b), $86 \mathrm{GHz}$ (c), and $129 \mathrm{GHz}$ (d), obtained on $1 \mathrm{March}$ 2016. The map peak fluxes are $2.39,2.41,1.84$, and $1.25 \mathrm{Jy} /$ beam at $22,43,86$, and $129 \mathrm{GHz}$. The lowest contour levels are $2.14,1.32,2.87$, and $7.51 \%$ of each map peak flux. Beam sizes are $5.79 \times 3.21,2.93 \times 1.58,1.47 \times 0.778$, and $0.972 \times 0.552$ at each frequency.

parameters including the total flux density $S_{\text {total }}$, the peak flux density $S_{\text {peak }}$, and the size $d$ of a circular Gaussian model, as listed in Table A.2. From the obtained model parameters, we estimated the brightness temperature $T_{\mathrm{b}}$ of core components using the following equation (Lee et al. 2008),

$T_{B}=\frac{2 \ln (2)}{\pi k} \frac{S_{\text {tot }} \lambda^{2}}{d^{2}}(1+z) \mathrm{K}$, where $k$ is the Boltzmann constant, $S_{\text {tot }}$ is the total flux density in Jy, $\lambda$ is the observing wavelength in $\mathrm{cm}, d$ is the estimated core size in radian, and $z$ is redshift. The estimated brightness temperatures are described in Table A.2 and scaled by $10^{10} \mathrm{~K}$. 
We derived uncertainties of each observable using the following equations (Fomalont 1999, Lee et al.|2008),

$$
\begin{aligned}
\sigma_{\text {peak }} & =\sigma_{\text {rms }}\left(1+\frac{S_{\text {peak }}}{\sigma_{\text {rms }}}\right)^{1 / 2}, \\
\sigma_{\text {total }} & =\sigma_{\text {peak }}\left(1+\frac{S_{\text {total }}^{2}}{S_{\text {peak }}^{2}}\right)^{1 / 2}, \\
\sigma_{d} & =d\left(\frac{\sigma_{\text {peak }}}{S_{\text {peak }}}\right)
\end{aligned}
$$

where these $\sigma_{\text {peak }}, \sigma_{\text {total }}$, and $\sigma_{d}$ are uncertainties of peak flux density, total flux density, and size, respectively. We regarded the maximum flux and residual noise in the region corresponding to the fitted Gaussian model as $S_{\text {peak }}$ and $\sigma_{\text {rms }}$.

\subsection{Multiwavelength Data}

We obtained publicly available data at $15 \mathrm{GHz}$ from the Owens Valley Radio Observatory (OVRO). The 1500 blazar monitoring program at $15 \mathrm{GHz}$ with the $40 \mathrm{~m}$ radio telescope at OVRO began in late 2007, approximately one year before the start of science operations of the Fermi-LAT (Richards et al. 2011). This monitoring is a high cadence with observations conducted approximately twice a week and with a typical flux uncertainty of $3 \%$. 4C +29.45 has been included in the OVRO sample since early 2008 .

In addition, we used $43 \mathrm{GHz}$ VLBI data from the VLBA-BU Blazar Monitoring Program (VLBA-BU-BLAZAR) ${ }^{1}$ This was obtained using the Very Long Baseline Array (VLBA). We used this data to estimate the size of emitting regions. The BU blazar group has an ongoing monitoring program which observes a total of $42 \gamma$-ray bright blazars at $43 \mathrm{GHz}$ (Jorstad \& Marscher. 2016). In this paper, in order to estimate the sizes of emitting regions within the source, we obtained the calibrated visibility (uv) data from the website and fitted the data with two-dimensional circular Gaussian models in a similar way as described in Section 2.3

\section{Results and Analysis}

\subsection{Multiwavelength Light Curves}

In order to study the multiwavelength behavior of the source, we compared the light curves at 22, 43, 86, and $129 \mathrm{GHz}$ from the iMOGABA observations with the OVRO (15 GHz) light curve. Although the $15 \mathrm{GHz}$ data are obtained from a single-dish radio telescope with a large beam size and the $22-129 \mathrm{GHz}$ data were obtained using VLBI observations with a small beam size, we can compare the trend of variability, assuming that the emitting region dominating the variability is compact at both scales. We compared the OVRO data with VLBI flux density obtained by VLBA (Lister et al.|2018), and we found the mean flux ratio between VLBI and single-dish is 0.9. Thus we decided to combine the VLBI and single-dish data for the time-series analysis. The combined multi-frequency light curves are shown in Fig. 2 .

\subsection{1. $15 \mathrm{GHz}$ Light Curve}

The $15 \mathrm{GHz}$ light curve of the source is shown in the top panel of Fig. 2. We used 211 epochs over the same period as the KVN observations from 5 December 2012 (MJD 56266) to 28 December

\footnotetext{
${ }^{1}$ https://www.bu.edu/blazars/VLBAproject.html
}

2016 (MJD 57750). The total flux density is in a range of $0.93-$ 2.42 Jy during the entire observing period. We divided the light curve into two periods, period A and period B. We defined period A as before MJD 56930 and period B as after MJD 56930. The flux density variability of the source is quite different before and after MJD 56930: the source was relatively quiet from MJD 56201 to MJD 56909 (period A). The flux density began to increase gradually after MJD 56930 (period B). Period A, the source flux density is in a range of 0.93-1.24 Jy with several minor enhancements. The mean flux density during the period $\mathrm{A}$ is $1.05 \mathrm{Jy}$ with a standard deviation of $0.07 \mathrm{Jy}$. Period B, the source became about twice as bright in about 110 days after MJD 56930, yielding a mean flux density during the period B of $1.85 \mathrm{Jy}$ with standard deviation of $0.35 \mathrm{Jy}$. Also, we found three flux enhancements around MJD 57100, 57300, and 57500 during the period B. The flux density increased from $1.01 \mathrm{Jy}$ to $2.42 \mathrm{Jy}$ in MJD 56900-57111, from 1.74 Jy to $2.01 \mathrm{Jy}$ in MJD 5722957282 , and from $1.78 \mathrm{Jy}$ to $2.35 \mathrm{Jy}$ in MJD 57416-57552.

\subsubsection{2-129 GHz Light Curves}

The KVN 22-129 GHz light curves are shown in panels (b)-(e) of Fig. 2. The total flux density is in a range of $0.72-2.88 \mathrm{Jy}$, 0.58-2.66 Jy, 0.26-1.93 Jy, and 0.27-1.26 Jy, at 22, 43, 86 and $129 \mathrm{GHz}$, respectively (Table A.1). The mean flux density (and its standard deviation) is $1.42 \mathrm{Jy}(0.62 \mathrm{Jy}), 1.28 \mathrm{Jy}(0.59 \mathrm{Jy})$, $0.97 \mathrm{Jy}(0.44 \mathrm{Jy}), 0.64 \mathrm{Jy}(0.26 \mathrm{Jy})$, at 22, 43, 86, and $129 \mathrm{GHz}$, respectively. Consistent with the OVRO light curve, we found that the KVN light curve at 22, 43, and $86 \mathrm{GHz}$ can be divided by two periods. The $129 \mathrm{GHz}$ light curve has fewer data points due to the limitation of the KVN system and we can not observe the same behavior as the lower frequencies.

\subsection{Variability Time Scales}

We investigated the variability time scales of the source by decomposing the $15 \mathrm{GHz}$ light curve using a number of combined exponential functions (e.g., Prince et al. 2017). There are several different forms of exponential equations. In Valtaoja \& Lainela (1999), they divided flares into two phases, rising and decaying. Both phases share the peak of the flare, and the ratio between rising and decaying time scales was set to be 1.3. In other words, the decaying time scales are 1.3 times longer than their corresponding rising time scales. On the other hand, Prince et al. (2017) combined both rising and decaying phases into one function, without fixing the ratio of their time scales. We followed the method in Prince et al. (2017).

We began by fitting the following function to the data (Prince et al. 2017),

$F(t)=\sum_{i=1}^{n} 2 F_{0, i}\left[\exp \left(\frac{t_{0, i}-t}{\tau_{r, i}}\right)+\exp \left(\frac{t-t_{0, i}}{\tau_{d, i}}\right)\right]^{-1}+b$.

Here, $n$ is the number of flares, and for individual flares, $F_{0, i}$ is the fitted local maximum flux density, $t_{0, i}$ is the time of the fitted maximum flux density, $\tau_{r, i}$ is the fitted rising time scale, $\tau_{d, i}$ is the fitted decaying time scale, and $b$ is a constant flux during our observational period. In order to determine the number of flares, $n$, to fit the light curve, we made a 30-day running average of the light curve and then defined a flare as being where the flux density was above the average. We have plotted the running average with the light curve in Fig. 3. The fitting algorithm requires initial conditions in order to constrain the fit. Where the flux density was above the average, we set the time of the local 


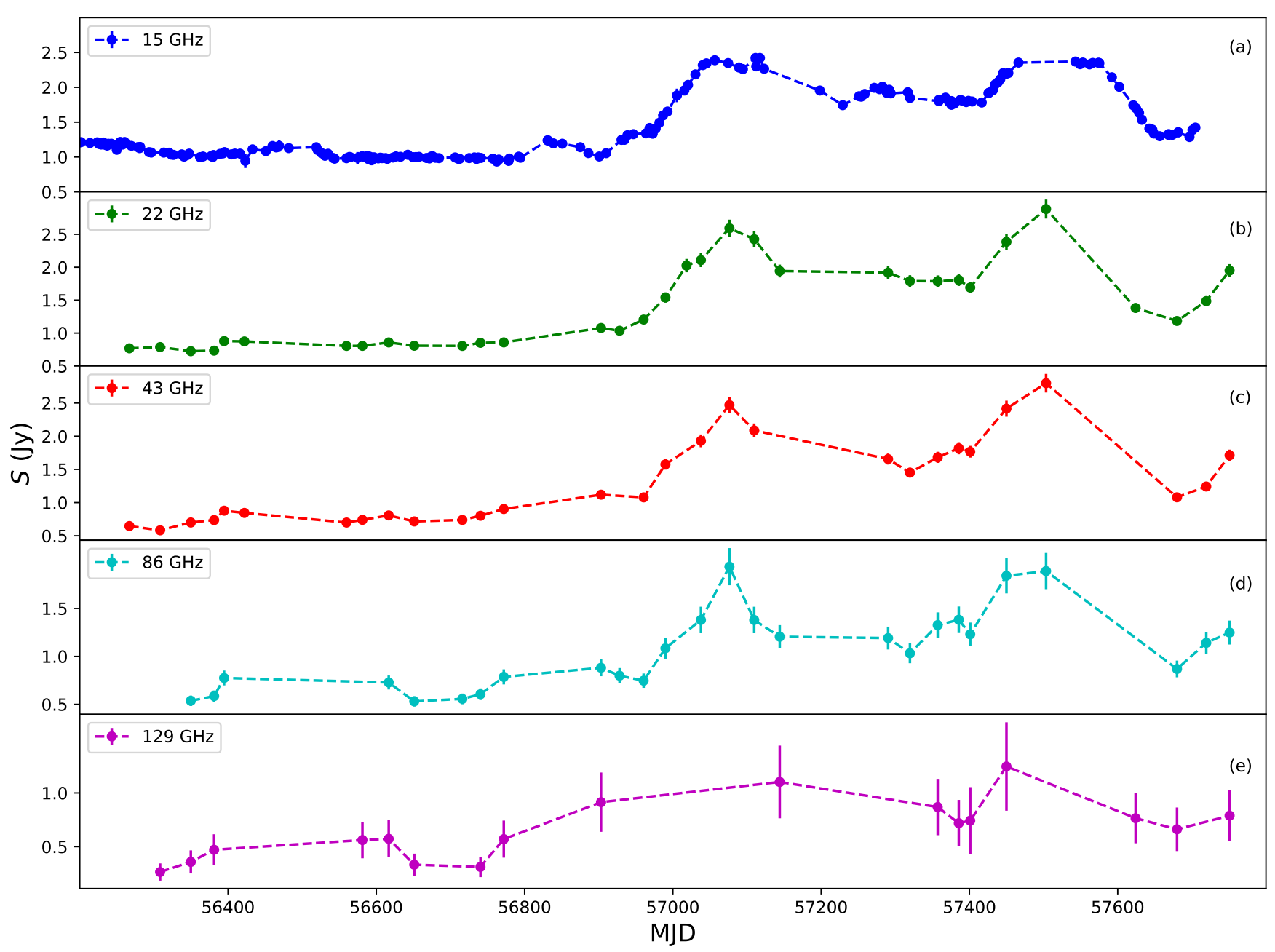

Fig. 2 Multiwavelength light curves. From the top to the bottom, data frequencies are 15 (obtained from OVRO), 22, 43, 86, 129 GHz (obtained from the KVN). Dashed lines are to connect the measurements.

peak and the flux density of that peak as the initial conditions for $t_{0, i}$ and $F_{0, i}$, respectively. Then we set the initial conditions for $\tau_{r, i}$ as the time between when the flux density began being above the average and then going below the average again. We found six flares $(n=6)$ in this way. We obtained the best fitting model with a total of 25 free parameters. The results of this fit are summarized in Table 1 . The results of the decomposition are shown in Fig. 4 and the reduced chi-squared of this fit was 8.4. It can be seen that the 4th flare potentially has additional structure, so we tried fitting with 2 functions to this flare. However, due to the lack of the data during the period of from MJD 57123 to 57198 , the additional flare seems to be constructed with large uncertainty. Therefore, we did not fit any additional functions, even the reduced chi-squared value has been improved. The first flare was not well constrained and we therefore excluded it from our analysis. In the last flare, there was a KVN $22 \mathrm{GHz}$ data point near the fitted peak from this analysis. The KVN data point has a value of $2.88 \mathrm{Jy}$ which compares well with the fitted values in Table 1. giving us confidence in the accuracy of the fit.
Table 1 Best fitting parameters from the decomposition of the OVRO $15 \mathrm{GHz}$ light curve.

\begin{tabular}{ccccc}
\hline \hline Flare & $\begin{array}{c}F_{0} \\
(\mathrm{Jy})\end{array}$ & $\begin{array}{c}\tau_{\mathrm{r}} \\
(\text { day })\end{array}$ & $\begin{array}{c}\tau_{\mathrm{d}} \\
(\text { day })\end{array}$ & $\begin{array}{c}t_{0} \\
(\mathrm{MJD})\end{array}$ \\
\hline 1 & $0.14 \pm 0.03$ & $187 \pm 136$ & $23 \pm 5$ & $56273 \pm 11$ \\
2 & $0.16 \pm 0.01$ & $62 \pm 6$ & $25 \pm 4$ & $56495 \pm 6$ \\
3 & $0.23 \pm 0.02$ & $9 \pm 4$ & $21 \pm 4$ & $56830 \pm 3$ \\
4 & $1.29 \pm 0.06$ & $40 \pm 2$ & $118 \pm 17$ & $57040 \pm 5$ \\
5 & $0.57 \pm 0.09$ & $40 \pm 10$ & $90 \pm 22$ & $57278 \pm 12$ \\
6 & $1.61 \pm 0.04$ & $57 \pm 5$ & $71 \pm 1$ & $57512 \pm 1$ \\
\hline
\end{tabular}

Notes. $F_{0}$ is the amplitude of each exponential function, $\tau_{r}$ is the rising time scale of each exponential function, and $t_{0}$ is the time of the local maximum of each exponential function. We obtained the best fitting model with 25 free parameters including a constant flux $b$ whose fitting result is $b=0.98 \mathrm{Jy}$.

\subsubsection{Physical parameters from the variability time scales}

Once we obtained the variability time scales, we were able to estimate the variability brightness temperatures and the variability Doppler factors. The Doppler factor can be estimated if there is a maximum intrinsic brightness temperature $\left(T_{\mathrm{B}}^{\text {int }}\right)$ that the source 


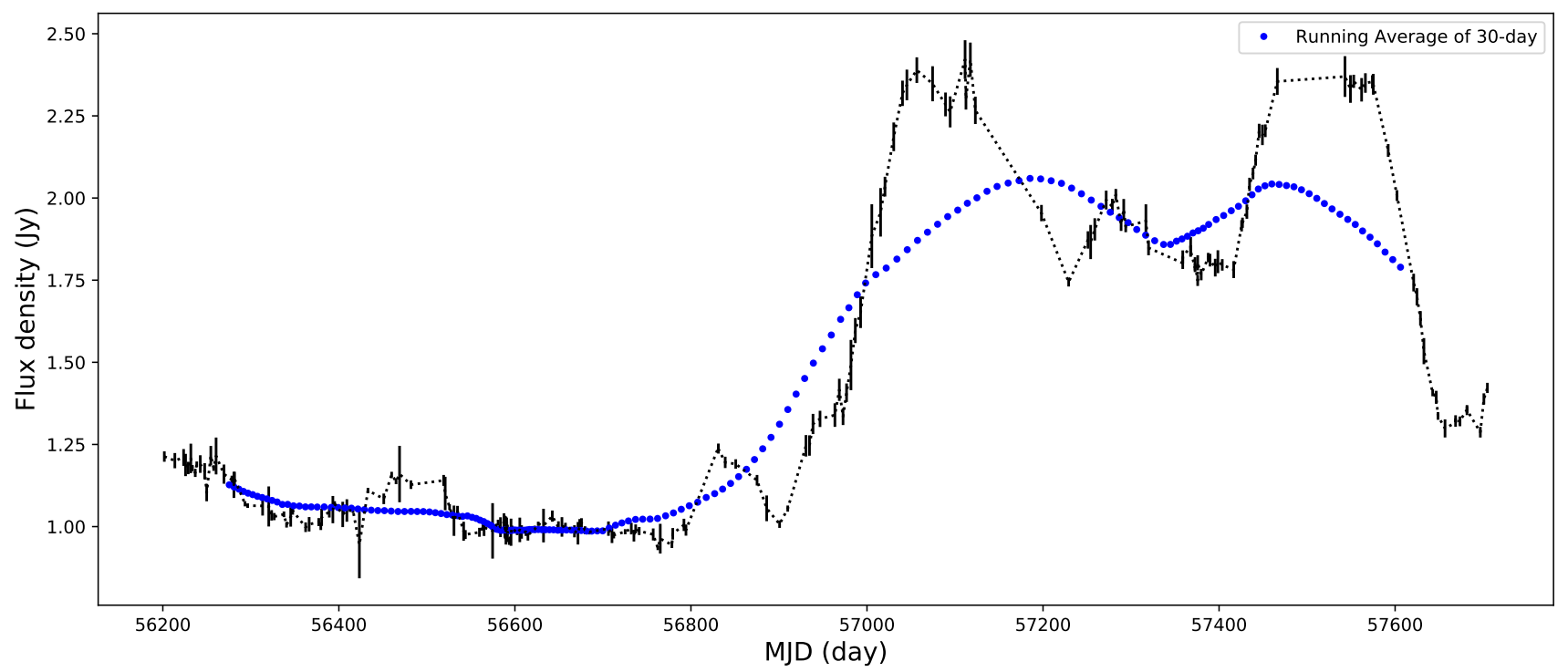

Fig. 3 30-day running average of the OVRO $15 \mathrm{GHz}$ data (blue). A flare was defined as when the light curve was above the running average. The times at which the flux density was above the average and the peak flux density within this time were used as initial conditions for the fitting algorithm described in Section 3.2. Dotted lines are to connect the measurements.

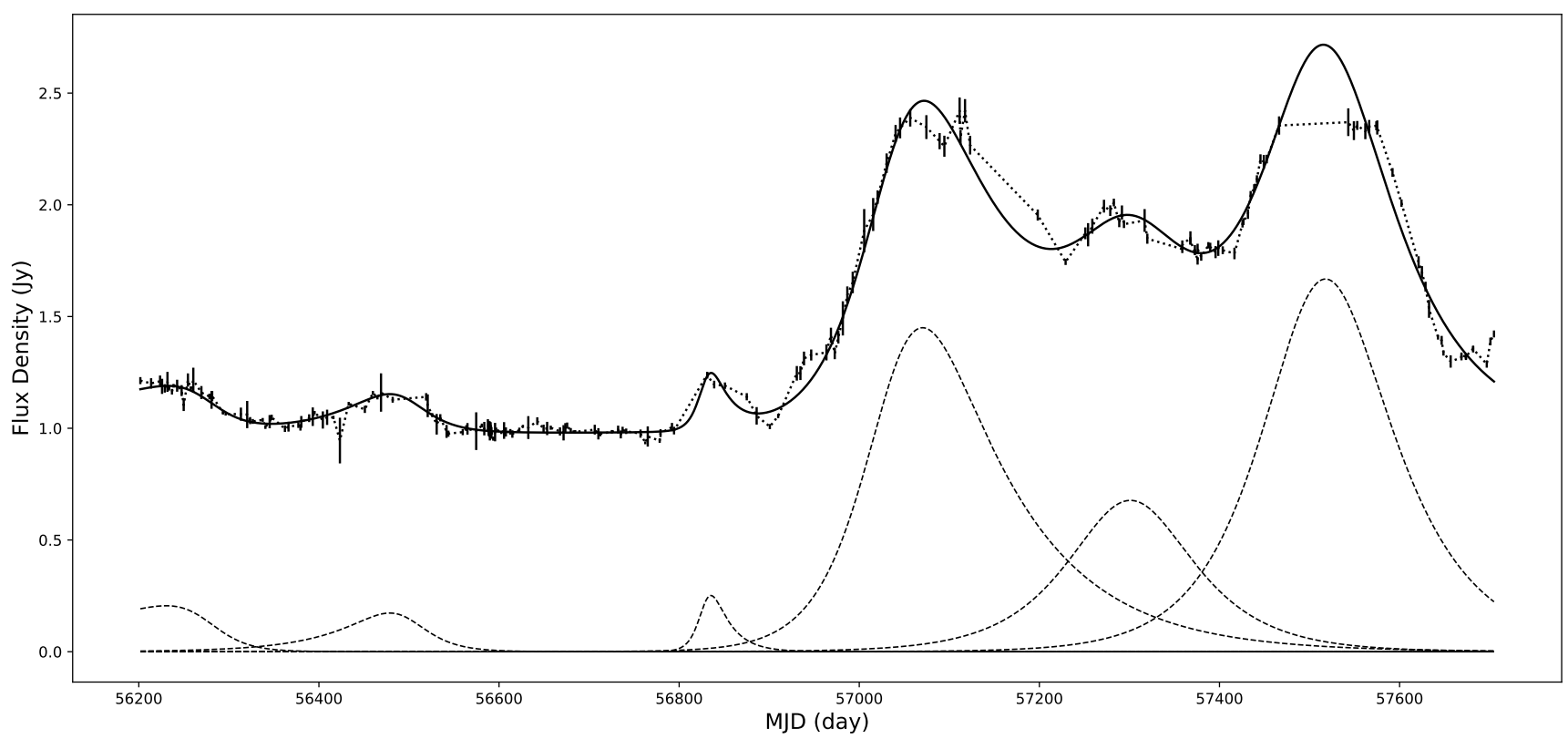

Fig. 4 Decomposition of the OVRO light curve with six flares. We could not constrain the peak for the first flare, we did not use the first flare to estimate the variability brightness temperature $T_{\mathrm{B}}^{\mathrm{var}}$ and the variability Doppler factor $\delta_{\mathrm{var}}$. Dotted line is to connect the measurements, dashed lines are the best fitting-models of individual flares, and solid line is the sum of the individual models.

can achieve. The Doppler factor is then proportional to the observed brightness temperature $\left(T_{\mathrm{B}}^{\mathrm{var}}\right)$ using the following equations (Fuhrmann et al.|2008, Rani et al. 2013):

$$
\begin{aligned}
T_{\mathrm{B}}^{\mathrm{var}} & =4.077 \times 10^{13}\left(\frac{D_{\mathrm{L}}}{v \tau_{\mathrm{r}, e}}\right)^{2} \frac{\Delta S}{(1+z)^{4}} \mathrm{~K}, \\
\delta_{\mathrm{var}} & =(1+z)\left(\frac{T_{\mathrm{B}}^{\mathrm{var}}}{T_{\mathrm{B}, \mathrm{eq}}}\right)^{1 / 3},
\end{aligned}
$$

where $\Delta S$ is the difference of flux density between the beginning and the peak of a flare, measured in Jy, $v$ is the observing fre- quency in $\mathrm{GHz}(15 \mathrm{GHz}), D_{L}=4477.8 \mathrm{Mpc}$ (assuming $\Omega_{m}=0.3$ and $\left.H_{0}=70 \mathrm{~km} / \mathrm{Mpc} / \mathrm{s}\right), \tau_{r}$ is time scale, estimated in day, and we assume that the $T_{\mathrm{B} \text {,eq }}$ is equal to $5 \times 10^{10} \mathrm{~K}$ (Hovatta et al. 2009). A detailed derivation of $T_{\mathrm{B}}^{\mathrm{var}}$ is explained in Appendix A In this estimation, we adopt an $e$-folding time scale $\tau_{\mathrm{r}, e}$, which is corresponding to the time between the peak of the flare and peak/e. Moreover, we set the flux difference between peak and peak/e of a flare as $\Delta S$. The estimated variability brightness temperatures and Doppler factors are listed in Table 2. 
Table 2 Estimated variability brightness temperatures and Doppler factors.

\begin{tabular}{ccc}
\hline \hline & $T_{\mathrm{b}}^{\mathrm{var}}$ & \\
Flare & $\left(10^{12} \mathrm{~K}\right)$ & $\delta_{\text {var }}$ \\
\hline 2 & $6.52 \pm 1.19$ & $8.77 \pm 0.53$ \\
3 & $192.97 \pm 78.22$ & $27.12 \pm 3.66$ \\
4 & $47.86 \pm 8.60$ & $17.04 \pm 1.02$ \\
5 & $23.65 \pm 9.23$ & $13.47 \pm 1.75$ \\
6 & $41.85 \pm 3.49$ & $16.29 \pm 0.45$ \\
\hline
\end{tabular}

Notes. In the analysis, we excluded the estimation for the first flare, because it appeared only decaying phase in our period.

In addition, we can estimate the Doppler factors from jet kinematics (e.g. apparent jet speed $\beta_{\text {app }}$ ) assuming the critical viewing angle $\left(\theta=\theta_{\text {crit }}\right)$ of the jet using the following equations;

$$
\begin{aligned}
\cos \theta_{\text {crit }} & =\beta \\
\sin \theta_{\text {crit }} & =\sqrt{1-\beta^{2}}=1 / \Gamma \\
\beta_{\text {app }} & =\frac{\beta \sin \theta}{1-\beta \cos \theta}=\frac{\beta}{\sqrt{1-\beta^{2}}}, \\
\delta & =\frac{1}{\Gamma(1-\beta \cos \theta)}=\frac{1}{\Gamma\left(1-\beta^{2}\right)}=\Gamma=\sqrt{1+\beta_{\text {app }}^{2}}
\end{aligned}
$$

In this estimation, we used the maximum apparent speed of $\beta_{\text {app }}=24.6 \pm 2.0$ obtained from Lister et al. (2019) assuming the jet viewing angle is at the critical angle, $\theta=\theta_{\text {crit }}$. We found that the Doppler factor at the critical angle is $\delta_{\text {crit }}=24.62$, which is comparable with the maximum variability Doppler factor of $\delta=27.12 \pm 3.66$ obtained for the flare 3, even though the observational periods are different.

\subsection{Cross-correlation Analysis}

In order to investigate a time lag of variations among the multifrequency light curves, we compared the $15 \mathrm{GHz}$ light curve with that of 22,43 , and $86 \mathrm{GHz}$, using a discrete cross-correlation function. In this analysis, we excluded the $129 \mathrm{GHz}$ data because they are very sparse in time and have relatively large errors. The KVN data obtained at 22, 43 , and $86 \mathrm{GHz}$ were compared to the OVRO $15 \mathrm{GHz}$ data in this analysis, yielding three data pairs as 15-22 GHz, 15-43 GHz, and 15-86 GHz. We used the unbinned discrete cross-correlation function (UDCF), in order to avoid interpolating and sampling errors (Edelson \& Krolik 1988), following

$$
\begin{aligned}
\operatorname{UDCF}_{i j} & =\frac{\left(a_{i}-\bar{a}\right)\left(b_{j}-\bar{b}\right)}{\sqrt{\left(\sigma_{a}^{2}-e_{a}^{2}\right)\left(\sigma_{b}^{2}-e_{b}^{2}\right)}}, \\
\operatorname{DCF}(\tau) & =\frac{1}{M} \mathrm{UDCF}_{i j} .
\end{aligned}
$$

Here, $a_{i}$ and $b_{j}$ are $i$ th and $j$ th observed data, $\bar{a}$ and $\bar{b}$ are mean values of the data sets $a$ and $b, \sigma_{a}$ and $\sigma_{b}$ are standard deviations of the data sets $a$ and $b$, and $e_{a}$ and $e_{b}$ are measurements errors, respectively. The UDCF is binned with a mean cadence of $\Delta \tau=$ 8 days and the number of data points $M$ in the bin, to estimate the DCF (equation 13). The statistical uncertainties of the time lag determined from the DCF analysis were obtained by performing a Monte-Carlo simulation based on a random subset selection method (Peterson et al. 1998; Lee et al. 2017). Samples of DCF
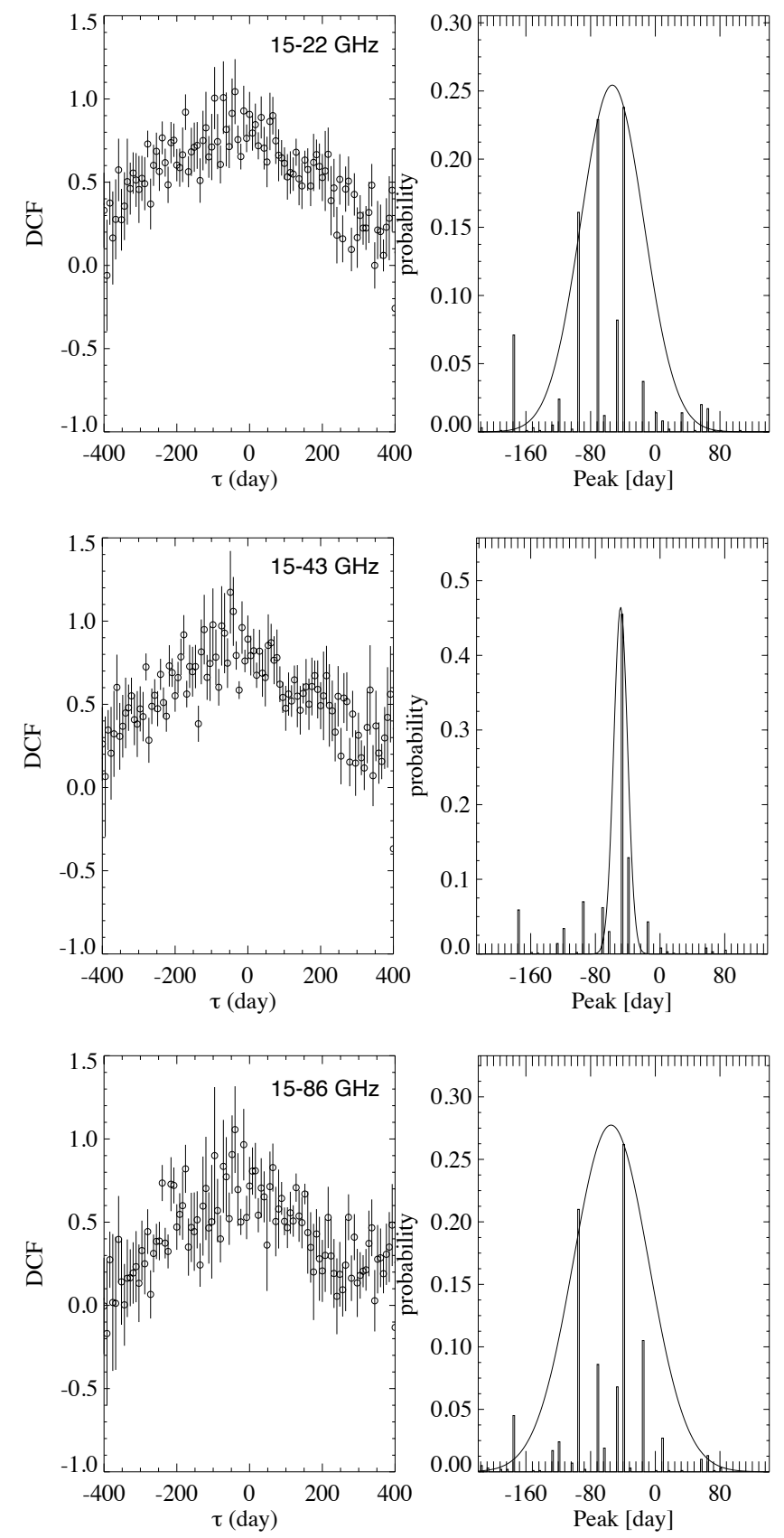

Fig. 5 Left: Samples of discrete cross-correlation functions of 15-22 GHz (top), 15-43 GHz (middle), and 15-86 GHz (bottom). Right: Cross-correlation peak distribution of the corresponding DCFs of 1000 iterations. We fitted the distribution with a Gaussian function in order to specify the mean value and adopt the standard deviation as an uncertainty.

are shown in the left panels of Fig. 5. A set of $N=1000$ light curves randomly sampled were used for determining the correlation peaks, yielding 1000 time delays and their distribution, as shown in the right panels of Fig. 5. By fitting the Gaussian function to the distribution, the statistical uncertainty of the time lag was determined. From the discrete cross-correlation analysis, we found the time lags of $-54 \pm 35,-50 \pm 9$, and $-55 \pm 47$ days at 22,43 , and $86 \mathrm{GHz}$ light curve from $15 \mathrm{GHz}$ light curve, respectively. We also obtained the confidence interval of $95 \%$ from -56 to $-52,-51$ to -49 , and -58 to -52 at $15-22,15-43$, and $15-$ 
$86 \mathrm{GHz}$, respectively (Lee et al. 2017). We performed this analysis for the whole period, since it is hard to distinguish the time lag of individual flares, finding that the radio flux enhancements at $22-86 \mathrm{GHz}$ lead that of $15 \mathrm{GHz}$ light curve. However it is hard to compare among $22-86 \mathrm{GHz}$, because the $\mathrm{KVN}$ observations were conducted simultaneously with the large cadence of about 30 days. Although we are not in a position to distinguish the time lag between 22 and $86 \mathrm{GHz}$ light curves, the fact that the high frequency $(22-86 \mathrm{GHz})$ light curves lead that of $15 \mathrm{GHz}$ light curve implies an opacity effect governing the multi-frequency (15 GHz vs $22 \mathrm{GHz}$ and higher) light curve.

\subsection{Turnover Frequency}

Synchrotron radiation originates from relativistic electrons traveling in a magnetic field and has an energy distribution of $N(E) \propto E^{-\delta}$, where $N(E)$ is the electron number density with its energy of $E$, has a power-law distribution as a function of frequency, $S \propto v^{\alpha}$, where $\alpha$ is the spectral index. Theoretically, synchrotron emission from an optically thin region has the spectral index of $\alpha=\left(1-\delta_{e}\right) / 2$, where $\delta_{e}$ is the Doppler factor of the relativistic electrons, while the emission from the optically thick region has the value of $\alpha=2.5$ (Rybicki \& Lightman 1979). In order to study the characteristics of the observed synchrotron emitting region, we calculated the spectral indices between each pair of adjacent frequencies. These results are listed in Table 3 , ranging from -0.4 to 0.1 at $22-43 \mathrm{GHz}$, from -0.6 to -0.1 at $43-86 \mathrm{GHz}$, and from -1.6 to 0.1 at $86-129 \mathrm{GHz}$. We found that the source is optically thinner at higher frequencies and has a flatter spectral index at lower frequencies.

Synchrotron emission can be absorbed by the same population of synchrotron particles (i.e., electrons) which have the same energy level at low frequencies (e.g., radio frequencies) in the optically thick region. This process is called SSA. Due to the absorption of the synchrotron radiation at lower frequencies, the synchrotron spectrum has a peak at a critical frequency, known as the turnover frequency, $v_{\mathrm{r}}$. This frequency is where the source transitions from being optically thick to optically thin. In order to determine the turnover frequency of the spectrum from the KVN multi-frequency observations, we fitted the observed spectra with the following curved power-law function (Algaba et al. $2018 \mathrm{~b}$, and references therein):

$S(v)=S_{\mathrm{r}}\left(\frac{v}{v_{\mathrm{r}}}\right)^{\alpha+b \ln \left(v / v_{r}\right)}$.

Here, $S(v)$ is the flux density at a given frequency, $v_{\mathrm{r}}$ is a arbitrary reference frequency, $\alpha$ is the spectral index, $S_{\mathrm{r}}$ is the flux density for when the $v$ is the same as $v_{r}$, and $b$ is a constant. Because we wish to find the turnover frequency, we set $\alpha$ to be zero. Among the 33 epochs, we selected 13 epochs with four-frequency measurements available and attempted to fit the spectra.

We assume that the CLEAN fluxes at four KVN frequencies originate from the core, a synchrotron self-absorbed region. However, in reality, an optically thin, extended jet can contribute to the CLEAN fluxes. The relative contribution of the jet compared with the core to the CLEAN fluxes becomes larger at lower frequencies, introducing possible bias to the data. Also, the limited uv-coverage of the KVN data may result in an artificial spectral steepening because short baselines could not be sampled at high frequencies (Kim et al. 2018a). We calculated an artificial spectral index of the source following Kim et al. (2018a). In this test, we produced simulated data to KVN array using the AIPS task UVCON with the Stokes I image FITS data
Table 3 Spectral indices from KVN data.

\begin{tabular}{|c|c|c|c|}
\hline 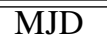 & $22-43 \mathrm{GHz}$ & 43-86 GHz & 86-129 GHz \\
\hline 56266 & -0.25 & - & - \\
\hline 56308 & -0.44 & - & - \\
\hline 56349 & -0.05 & -0.38 & -1.02 \\
\hline 56381 & 0.01 & -0.33 & -0.49 \\
\hline 56394 & 0.00 & -0.18 & - \\
\hline 56422 & -0.05 & - & - \\
\hline 56559 & -0.20 & - & - \\
\hline 56581 & -0.12 & - & - \\
\hline 56616 & -0.09 & -0.15 & -0.59 \\
\hline 56651 & -0.17 & -0.43 & -1.11 \\
\hline 56716 & -0.13 & -0.41 & - \\
\hline 56740 & -0.09 & -0.40 & -1.64 \\
\hline 56771 & 0.07 & -0.20 & -0.78 \\
\hline 56903 & 0.06 & -0.34 & 0.11 \\
\hline 56960 & -0.16 & -0.53 & - \\
\hline 56989 & 0.03 & -0.54 & - \\
\hline 57037 & -0.13 & -0.48 & - \\
\hline 57076 & -0.07 & -0.35 & - \\
\hline 57109 & -0.22 & -0.60 & - \\
\hline 57144 & - & - & -0.17 \\
\hline 57290 & -0.21 & -0.48 & - \\
\hline 57319 & -0.30 & -0.49 & - \\
\hline 57357 & -0.09 & -0.34 & -1.05 \\
\hline 57385 & 0.01 & -0.40 & -1.63 \\
\hline 57401 & 0.06 & -0.52 & -0.42 \\
\hline 57449 & 0.02 & -0.39 & -0.74 \\
\hline 57503 & -0.04 & -0.57 & - \\
\hline 57680 & -0.13 & -0.31 & -0.63 \\
\hline 57719 & -0.26 & -0.12 & - \\
\hline 57750 & -0.19 & -0.46 & -1.15 \\
\hline
\end{tabular}

Notes. These spectral indices were estimated among three pairs from 22 to $129 \mathrm{GHz}$ linearly regarding single power-law spectrum. The errors are $0.10,0.16$ and 0.79 for the pairs of $22-43,43-86$ and $86-129 \mathrm{GHz}$, respectively.

(for epoch of 2012 November 16), obtained from the MOJAVE website ${ }^{2}$ We found that the artificial spectral index of the source is 0.04 , implying that the artificial spectral steepening due to the limited uv-coverage of the KVN data is negligible. We also considered additional uncertainty of $30 \%$, which is obtained from a mean difference between KVN CLEAN flux density and BU core flux density selected to have a time difference within 7 days at $43 \mathrm{GHz}$.

We obtained turnover frequencies at 12 epochs excluding epoch 16 (MJD 56903) when the fit was failed. The fitting results are shown in Fig. 6 and summarized in Table 4 . We found that $4 \mathrm{C}+29.45$ has a turnover frequency ranging from $23 \mathrm{GHz}$ to $38 \mathrm{GHz}$ during MJD 56381-57750. Since the turnover frequency was measured to be below $43 \mathrm{GHz}$ in all epochs when a successful fit was obtained, based on the curved-power-law fitting analysis. we estimated the optically thin spectral index, $\alpha_{\text {thin }}$, by performing a linear fit to the flux density at 43,86 , and $129 \mathrm{GHz}$. We found these to be ranging from -0.74 to -0.25 and the results are shown in the Table 4

${ }^{2}$ http://www.physics.purdue.edu/astro/MOJAVE/allsources.html 

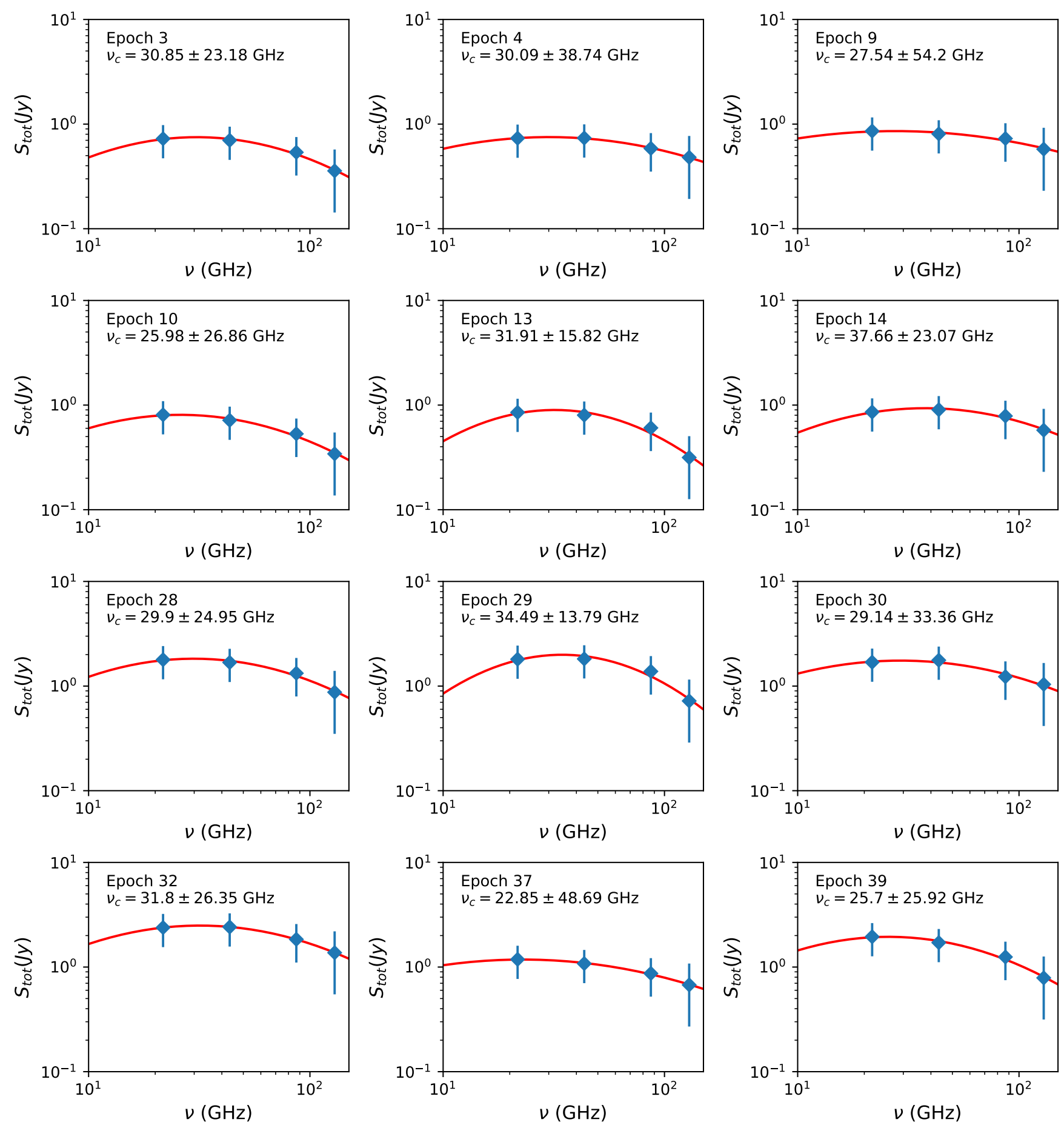

Fig. 6 The fitting results of turnover frequencies from KVN 4-frequency simultaneous data. The resulting turnover frequencies lie in the range of $\sim 23-38 \mathrm{GHz}$.

\subsection{Magnetic Field Strength}

From the obtained observational parameters, we can estimate the magnetic field strength of a synchrotron self-absorption region using the following equation (Marscher 1983; Hodgson et al. 2017);

$B_{\mathrm{SSA}}=10^{-5} b(\alpha) S_{\mathrm{r}}^{-2} \theta_{\mathrm{r}}^{4} \nu_{\mathrm{r}}^{5}\left(\frac{\delta}{1+z}\right)^{-1}$
Here, $b(\alpha)$ is a factor depending on the spectral index (refer to Table 1 in Marscher 1983). In order to provide a better estimate of $b(\alpha)$ than given in (Marscher 1983), we linearly interpolated the values listed in Table A.1 in Marscher (1983), and fit for the optically thin spectral indices found in Section 3.4 .

In order to estimate the size of emission region, $\theta_{\mathrm{r}}$, at the turnover frequency, we, first, obtained the $43 \mathrm{GHz}$ images of the source from the BU program that was observed both before and after the epochs where the turnover frequency could 
Table 4 The turnover frequency fitting results.

\begin{tabular}{cccc}
\hline \hline $\begin{array}{c}\text { MJD } \\
(\text { day })\end{array}$ & $\begin{array}{c}v_{\mathrm{r}} \\
(\mathrm{GHz})\end{array}$ & $\begin{array}{c}S_{\mathrm{r}} \\
(\mathrm{Jy})\end{array}$ & $\begin{array}{c}\alpha_{\text {thin }} \\
(1)\end{array}$ \\
$(2)$ & $(3)$ & $(4)$ \\
\hline 56349 & $30.85 \pm 23.18$ & $0.75 \pm 0.16$ & $-0.55 \pm 0.54$ \\
56381 & $30.09 \pm 38.74$ & $0.75 \pm 0.16$ & $-0.37 \pm 0.56$ \\
56616 & $27.54 \pm 54.20$ & $0.86 \pm 0.19$ & $-0.26 \pm 0.55$ \\
56651 & $25.98 \pm 26.86$ & $0.81 \pm 0.19$ & $-0.60 \pm 0.54$ \\
56740 & $31.91 \pm 15.82$ & $0.90 \pm 0.19$ & $-0.74 \pm 0.53$ \\
56771 & $37.66 \pm 23.07$ & $0.93 \pm 0.22$ & $-0.35 \pm 0.54$ \\
57357 & $29.90 \pm 24.95$ & $1.83 \pm 0.39$ & $-0.52 \pm 0.54$ \\
57385 & $34.49 \pm 13.79$ & $1.99 \pm 0.44$ & $-0.73 \pm 0.53$ \\
57401 & $29.14 \pm 33.63$ & $1.75 \pm 0.39$ & $-0.50 \pm 0.57$ \\
57449 & $31.80 \pm 26.35$ & $2.49 \pm 0.55$ & $-0.48 \pm 0.55$ \\
57680 & $22.85 \pm 48.69$ & $1.18 \pm 0.32$ & $-0.39 \pm 0.55$ \\
57750 & $25.70 \pm 25.92$ & $1.94 \pm 0.45$ & $-0.64 \pm 0.54$ \\
\hline
\end{tabular}

Notes. (1) Modified Julian Date, (2) estimated turnover frequencies, (3) maximum flux densities and (4) spectral indices of optically thin region (43-129 GHz from KVN data).

be measured. We then performed a simple linear interpolation in between these two VLBA epochs in order to determine the size of the $43 \mathrm{GHz}$ core at the epochs where the turnover frequency could be measured. The observed core size is expected to vary as a function of frequency, following the relationship

$\theta \propto v^{-\epsilon}$

where $\epsilon$ represents the geometry of the jet. If the jet is conical, $\epsilon=1$, and if the jet is parabolic, $\epsilon=0.5$ (e.g., Algaba et al. 2017). In this case $\epsilon$ was found to be $0.44 \pm 0.08$ by Algaba et al. (2017). Upper limits of the core size are obtained for 3 epochs, taking into account a minimum resolvable size (e.g., Lee et al. 2016b). All the uncertainties were obtained using standard error propagation method, and our derived expressions for calculating the uncertainties are given in appendix C. Moreover, we can estimate the emission region size $\theta_{\mathrm{r}}$ from the variability size $\theta_{\text {var }}$ (Hodgson et al.2020),

$\theta_{\mathrm{var}}=2(1+z) \frac{c \cdot \tau_{\mathrm{r}, e}}{D_{\mathrm{L}}} \delta_{\mathrm{var}}$.

In this analysis, we used the variability time scales $\tau_{\mathrm{r}, e}$ and the variability Doppler factors $\delta_{\text {var }}$ obtained in Section 3.2 Estimated variability sizes are listed in Table 6. Then we extrapolated these variability sizes at turnover frequencies $\left(\theta_{\mathrm{var}, v_{\mathrm{r}}}\right)$, taking into account the jet geometry $\epsilon$, in order to compare the sizes $\left(\theta_{\mathrm{VLBI}, v_{\mathrm{r}}}\right)$ extrapolated from $\theta_{\mathrm{VLBI}}$. By comparing those two sizes, we found that difference between those sizes is not significant (see Section 4.1). Therefore, we adopted the mean size between $\theta_{\mathrm{var}, v_{\mathrm{r}}}$ and $\theta_{\mathrm{VLBI}, v_{\mathrm{r}}}$ regarding the uncertainty as the minimum and maximum of their uncertainties. When the variability sizes is not available we used the extrapolated core sizes $\theta_{\mathrm{VLBI}, v_{\mathrm{r}}}$ (see Section 4.1). Our estimated core sizes at the turnover frequency are listed in Table 5.

It should be noted that for the factor $\frac{\delta}{(1+z)}$ in equation $\sqrt{15}$, we use a power index of -1 , instead of +1 as in Marscher (1983), because we consider that the observables originated from the core region which is assumed to be relatively stationary in time (Lee et al. 2017, Algaba et al. 2018b). We adopted $\delta=9.6 \pm 2.6$ for the source as obtained by Jorstad et al. (2017).

Therefore, we obtained 7 measurements ranging from $0.001_{-0.001}^{+0.003} \mathrm{G}$ to $0.099_{-0.098}^{+0.516} \mathrm{G}$. For the other epochs, only upper limits could be derived. In this estimation, we exclude epoch 3 (MJD 56349) because the observation was conducted during the first flare which is hard to constrain the rising time scale (3.2). The results of this analysis are shown in Table 5 and Fig. 7 .

In addition to the magnetic field strength $B_{\mathrm{SSA}}$ of a synchrotron self-absorption region, we can also estimate the minimum magnetic field strength $B_{\text {eq }}$ assuming equipartition between magnetic field and particle energy densities. The expression to describe this is as follows (Kataoka \& Stawarz. 2005. Algaba et al.2018a):

$$
\begin{aligned}
B_{\text {eq }} & = & 123 \eta^{2 / 7}(1+z)^{11 / 7}\left(\frac{D_{\mathrm{L}}}{100 \mathrm{Mpc}}\right)^{-2 / 7}\left(\frac{v_{\mathrm{r}}}{5 \mathrm{GHz}}\right)^{1 / 7} \\
& \times & \left(\frac{S_{\mathrm{r}}}{100 \mathrm{mJy}}\right)^{2 / 7}\left(\frac{\theta_{\mathrm{r}}}{0.3^{\prime \prime}}\right)^{-6 / 7} \delta^{-5 / 7} .
\end{aligned}
$$

Here, $\eta$ is a ratio between the energies of hadrons and leptons. $\eta$ is 1 for a leptonic model, and $\eta$ is 1836 for a hadronic model. In this analysis, we adopted a compromise value of $\eta \approx 100$ because we do not know the true composition of the jet. For the same reasons as before, the equipartition magnetic field strength could only be determined for 8 epochs. The equipartition magnetic field strengths were found to be ranging from $0.10 \pm 0.03 \mathrm{G}$ to $0.25 \pm 0.07 \mathrm{G}$. These values are largely up to two orders of magnitudes stronger than $B_{\mathrm{SSA}}$, except for two epochs (MJD 56616 and MJD 56651) where $B_{\text {SSA }}$ and $B_{\text {eq }}$ are approximately comparable within uncertainties. The results are shown in Table 5 and Fig. 7 .

Magnetic field strength of a core region in the jet can be estimated from a core-shift effect as described in Lobanov (1998) and Pushkarev et al. (2012). Assuming equipartition between particle and magnetic field energy density, spectral index of $\alpha=-0.5\left(S_{v} \propto v^{\alpha}\right)$ and a critical angle $\theta \approx \Gamma^{-1}$, the magnetic field strength at a distance of $1 \mathrm{pc}$ from the central engine is given by:

$B_{1} \simeq 0.04 \Omega_{r v}^{3 / 4}(1+z)^{1 / 2}\left(1+\beta_{\mathrm{app}}^{2}\right)^{1 / 8}$ Gauss,

where $z$ is redshift, $\beta_{\text {app }}$ is apparent jet speed and $\Omega_{r v}$ is the core shift measure defined in Lobanov (1998) as

$\Omega_{r v}=4.85 \times 10^{-9} \frac{\Delta r_{c} D_{\mathrm{L}}}{(1+z)^{2}} \frac{v_{1} v_{2}}{v_{2}-v_{1}}$

where $\Delta r_{c}$ is the core shift between different frequencies $v_{1}$ and $v_{2}\left(v_{1}<v_{2}\right)$. The magnetic field strength $B_{\mathrm{c}}$ at a core region $r_{\mathrm{c}}$ observed by VLBI observations can be estimated as $B_{\mathrm{c}}=B_{1} r_{\mathrm{c}}^{-1}$. The distance $r_{\mathrm{c}}$ of the core from the central engine is given by Lobanov (1998) as

$r_{c}(v)=\frac{\Omega_{r v}}{v \sin \theta} \approx \frac{\Omega_{r v}\left(1+\beta_{\mathrm{app}}^{2}\right)^{1 / 8}}{v} \mathrm{pc}$,

where $v$ is a given frequency. From the study on the core shift variability of the relativistic jets (Plavin et al. 2019), we found that $4 \mathrm{C}+29.45$ had a core shift of 1.02 mas measured at $v_{1}=2.3 \mathrm{GHz}$ and $v_{2}=6.8 \mathrm{GHz}$ on 2012 December 5 (MJD 56266), which is the closest measurement in time to our analysis. The core shift gives us a core shift measure of $\Omega_{r v}=23.4 \mathrm{pc} \mathrm{GHz}$ and magnetic field strength at $1 \mathrm{pc}$ of $B_{1}=1.25 \mathrm{G}$ taking into account the apparent speed of the jet $\beta_{\text {app }}=24.6$ (Lister et al. 2019). In order to compare the magnetic field strength $B_{\mathrm{c}}$ from the core shift to $B_{\mathrm{SSA}}$ of synchrotron self-absorption region, we estimated the core distance $r_{\mathrm{c}}\left(v_{\mathrm{r}}\right)=19.14 \mathrm{pc}$ at a turnover frequency of $v_{r}=30.09 \mathrm{GHz}$ (measured on MJD 56381). We found that the magnetic field strength $B_{\mathrm{c}}$ of the core region is $B_{\mathrm{c}}=0.065 \mathrm{G}$ which is an order of magnitude higher than $B_{\mathrm{SSA}}=0.006 \mathrm{G}$ on MJD 56381 . 


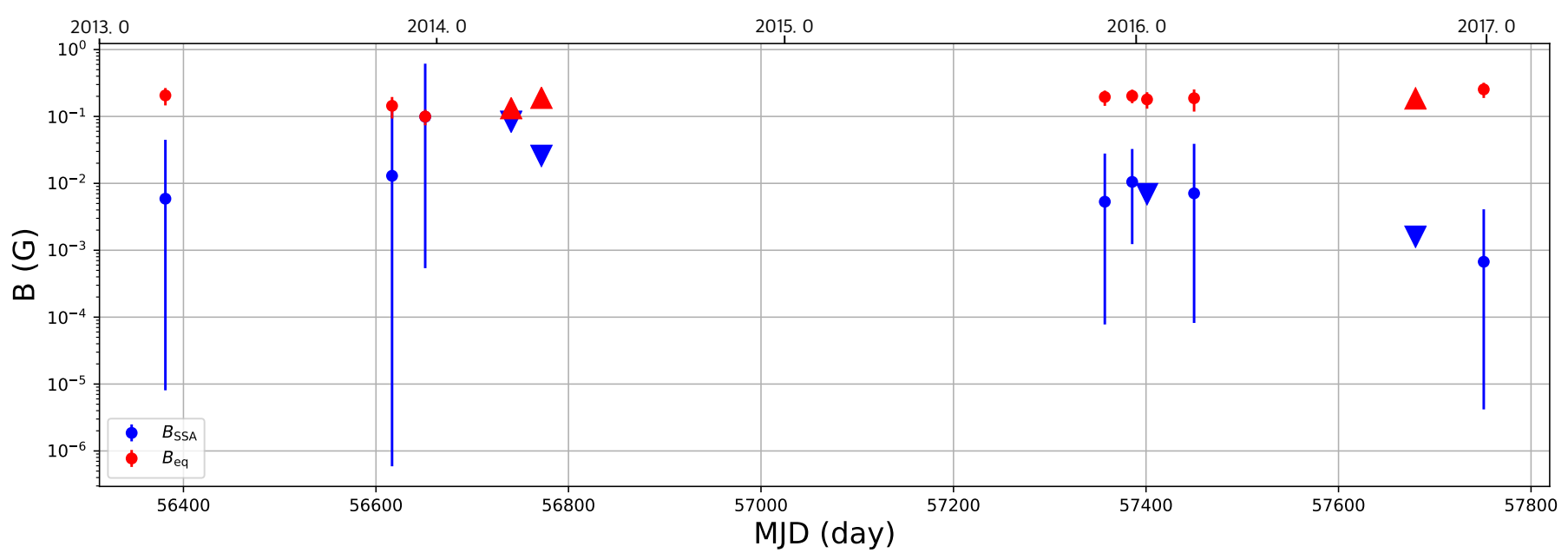

Fig. 7 Estimated magnetic field strengths of synchrotron self-absorption region and energy equipartition region. Red filled circles represent $B_{\text {eq }}$, while the blue filled circles represent $B_{\mathrm{SSA}}$. Triangles are lower limits and inverted triangles are upper limits. The lower uncertainty of $B_{\mathrm{SSA}}$ is computed in logarithm (see appendix C).

Table 5 Estimated B-Field Strengths of synchrotron self-absorption regions.

\begin{tabular}{ccccccc}
\hline \hline MJD & $b(\alpha)$ & $\begin{array}{c}S_{\mathrm{r}} \\
(\mathrm{Jy})\end{array}$ & $\begin{array}{c}\theta_{\mathrm{r}} \\
(\mathrm{mas})\end{array}$ & $\begin{array}{c}v_{\mathrm{r}} \\
(\mathrm{GHz})\end{array}$ & $\begin{array}{c}B_{\mathrm{SSA}} \\
(\mathrm{G})\end{array}$ & $\begin{array}{c}B_{\mathrm{eq}} \\
(\mathrm{G})\end{array}$ \\
\hline 56381 & $2.47 \pm 3.12$ & $0.75 \pm 0.16$ & $0.041_{-0.004}^{+0.005}$ & $30.09 \pm 38.74$ & $0.006_{-0.006}^{+0.039}$ & $0.206_{-0.060}^{+0.059}$ \\
56616 & $1.84 \pm 3.06$ & $0.86 \pm 0.19$ & $0.065_{-0.005}^{+0.005}$ & $27.54 \pm 54.20$ & $0.013_{-0.013}^{+0.130}$ & $0.144_{-0.051}^{+0.051}$ \\
56651 & $3.37 \pm 0.86$ & $0.81 \pm 0.19$ & $0.096_{-0.007}^{+0.007}$ & $25.97 \pm 26.86$ & $0.099_{-0.098}^{+0.516}$ & $0.100_{-0.026}^{+0.026}$ \\
56740 & $3.58 \pm 0.84$ & $0.90 \pm 0.19$ & $<0.074$ & $31.91 \pm 15.82$ & $<0.084$ & $>0.132$ \\
56771 & $2.35 \pm 3.04$ & $0.93 \pm 0.22$ & $<0.051$ & $37.66 \pm 23.07$ & $<0.026$ & $>0.189$ \\
57357 & $3.24 \pm 0.86$ & $1.83 \pm 0.39$ & $0.059_{-0.005}^{+0.008}$ & $29.90 \pm 24.95$ & $0.005_{-0.005}^{+0.023}$ & $0.195_{-0.051}^{+0.048}$ \\
57385 & $3.57 \pm 0.84$ & $1.99 \pm 0.44$ & $0.060_{-0.008}^{+0.005}$ & $34.49 \pm 13.79$ & $0.011_{-0.009}^{+0.022}$ & $0.203_{-0.045}^{+0.048}$ \\
57401 & $3.2^{*}$ & $1.75 \pm 0.39$ & $0.064_{-0.008}^{+0.006}$ & $29.14 \pm 33.36$ & $<0.007$ & $0.180_{-0.049}^{+0.051}$ \\
57449 & $3.07 \pm 3.08$ & $2.49 \pm 0.55$ & $0.070_{-0.021}^{+0.023}$ & $31.80 \pm 26.35$ & $0.007_{-0.007}^{+0.032}$ & $0.187_{-0.069}^{+0.066}$ \\
57680 & $2.59 \pm 3.09$ & $1.18 \pm 0.32$ & $<0.052$ & $22.85 \pm 48.69$ & $<0.002$ & $>0.185$ \\
57750 & $3.42 \pm 0.86$ & $1.95 \pm 0.45$ & $0.043_{-0.003}^{+0.003}$ & $25.70 \pm 25.72$ & $0.001_{-0.001}^{+0.003}$ & $0.254_{-0.065}^{+0.065}$ \\
\hline
\end{tabular}

Notes. The estimated magnetic field strength and parameters.

\section{Discussion}

\subsection{Testing Our Assumptions on the Size Estimates}

According to Eq. 15 for estimating $B_{\mathrm{SSA}}$, the emission size $\theta_{\mathrm{r}}$ is one of the important factors for $B_{\mathrm{SSA}}$ estimates $\left(B_{\mathrm{SSA}} \propto \theta_{\mathrm{r}}^{4}\right)$, and hence the uncertainty of the emission size. The determination of the emission size $\theta_{\mathrm{r}}$ presented in Section 3.5 rely on two assumptions. Firstly, the variability size (e.g., $\theta_{\text {var }}$ ), in principle, is an intrinsic size of variable emission region (e.g., a VLBI core) and a high resolution VLBI observation may be able to resolve the emission region size (e.g., $\left.\theta_{\mathrm{VLBI}}\right)$. Therefore, at a given frequency $v$, those sizes are comparable $\left(\theta_{\mathrm{var}, v} \approx \theta_{\mathrm{VLBI}, v}\right)$ if the VLBI observations can resolve the emission region. Secondly, a jet transverse radius $R$ (or a jet angular size $\theta$ ) expands along the distance $r$ of a VLBI core from the central engine $\left(R \propto r^{\epsilon}\right.$ or $\left.\theta \propto r^{\epsilon}\right)$ and the distance of the core (i.e., optical depth $\tau=1$ surface in the jet) depends on the observing frequency $v\left(r \propto v^{-1}\right)$. Therefore, the jet geometry assumption $\theta \propto v^{-\epsilon}$ used to extrapolate the sizes to the turnover frequency is valid.

For such a case that we have a variability size $\theta_{\text {var }}$ measured at a frequency $v_{1}$ and a VLBI size $\theta_{\mathrm{VLBI}}$ observed at another frequency $v_{2}$, we then expect that extrapolated sizes $\theta_{\mathrm{var}, v_{3}}$ and $\theta_{\mathrm{VLBI}, v_{3}}$ at a common frequency $v_{3}$ are comparable. In this study, we obtained $\theta_{\text {VLBI }}$ from VLBA observations at $43 \mathrm{GHz}$ and $\theta_{\text {var }}$ from the variability time scales at $15 \mathrm{GHz}$. In order to check these assumptions, we extrapolated the sizes at turnover frequencies based on the jet geometry $\epsilon=0.44$. We found that the esti-

Table 6 Size comparison between VLBI measurement and variability.

\begin{tabular}{ccccc}
\hline \hline $\begin{array}{c}\text { MJD } \\
(1)\end{array}$ & $\begin{array}{c}\theta_{\mathrm{var}, 15 \mathrm{GHz}} \\
\text { (mas) }\end{array}$ & $\begin{array}{c}\theta_{\mathrm{var}, v_{\mathrm{r}}} \\
(\mathrm{mas})\end{array}$ & $\begin{array}{c}\theta_{\mathrm{VLBI}, v_{\mathrm{r}}} \\
(\mathrm{mas})\end{array}$ & $\begin{array}{c}\theta_{\mathrm{VLBI}, 43 \mathrm{GHz}} \\
(\mathrm{mas})\end{array}$ \\
\hline 56381 & $0.055 \pm 0.002$ & $0.040 \pm 0.003$ & $0.043 \pm 0.003$ & $0.036 \pm 0.003$ \\
57357 & $0.083 \pm 0.007$ & $0.061 \pm 0.006$ & $0.057 \pm 0.003$ & $0.049 \pm 0.002$ \\
57385 & $0.083 \pm 0.007$ & $0.058 \pm 0.006$ & $0.061 \pm 0.003$ & $0.056 \pm 0.003$ \\
57401 & $0.083 \pm 0.007$ & $0.062 \pm 0.006$ & $0.066 \pm 0.004$ & $0.056 \pm 0.003$ \\
57449 & $0.122 \pm 0.002$ & $0.088 \pm 0.005$ & $0.051 \pm 0.003$ & $0.045 \pm 0.002$ \\
\hline
\end{tabular}

Notes. (1) Date close to flare 2-6, (2) variability size estimated from the variability time scale at $15 \mathrm{GHz},(3)$ variability size at turnover frequency $v_{r}$ extrapolated from the variability size at $15 \mathrm{GHz}, \theta_{\mathrm{var}, 15 \mathrm{GHz}}$, using the jet geometry of $\epsilon=0.44$, (4) the extrapolated VLBI size at turnover frequency from the VLBI size at $43 \mathrm{GHz}$, and (5) the size directly determined by VLBI observations at $43 \mathrm{GHz}$ using the VLBI data from the VLBA-BU-Blazar monitoring program (Jorstad et al. 2017). 


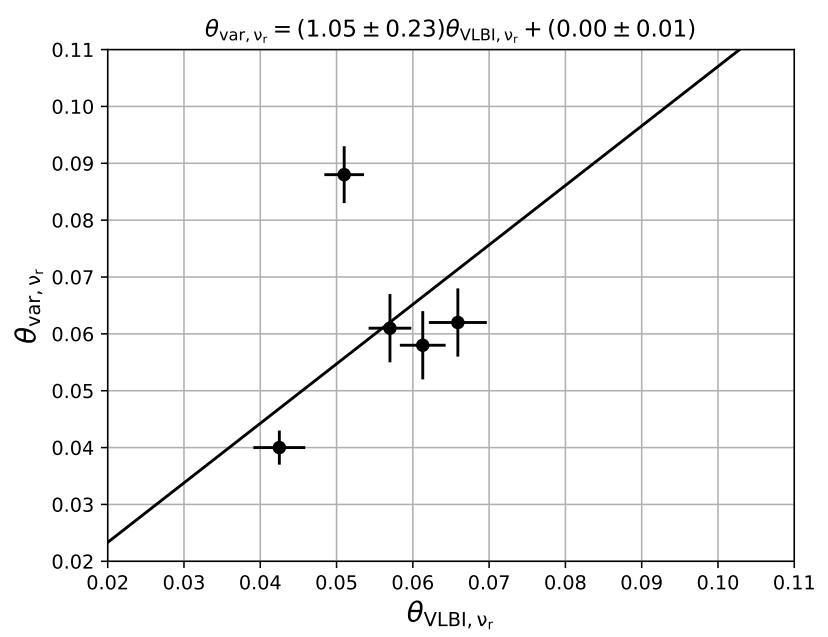

Fig. 8 Comparison between extrapolated sizes $\theta_{\mathrm{var}, v_{\mathrm{r}}}$ and $\theta_{\mathrm{VLBI}, v_{r}}$ at turnover frequency from $\theta_{\mathrm{var}, 15}$ and $\theta_{\mathrm{VLBI}, 43}$ based on the jet geometry $\epsilon=0.44$ (Algaba et al.2017). The solid line represents the result of the linear fitting (as also described in top of the figure).

mated variability sizes were generally comparable with the sizes observed directly by VLBI observations as shown in Figure 8 . In order to specify the differences between $\theta_{\mathrm{var}, v_{\mathrm{r}}}$ and $\theta_{\mathrm{VLBI}, v_{\mathrm{r}}}$, we performed a linear fit to the data for 5 epochs (Fig. 8). The fitting result shows a slope of $1.05 \pm 0.23$ between $\theta_{\mathrm{var}, v_{\mathrm{r}}}$ and $\theta_{\mathrm{VLBI}, \nu_{\mathrm{r}}}$, even though one data point for one epoch does not follow the trend. This implies that the assumptions on the size estimation are valid. Therefore, we decided to use the mean value of two sizes taking into the uncertainties. However, for the other 6 epochs, we decided to use the $\theta_{\mathrm{VLBI}, \nu_{\mathrm{r}}}$, because it is hard to constrain the correlation due to the time gap between those two sizes.

\subsection{Brightness Temperature}

We obtained the brightness temperatures from the KVN data (Table A.2). The median brightness temperatures obtained from model fitting the KVN data are shown in Table A.2. We found a significant $(>3 \sigma)$ trend of decreasing brightness temperature with increasing frequency. In the previous studies analyzing KVN observations, we see increasing brightness temperatures with increasing observing frequency because the beam size decreases with increasing observing frequency (Lee et al. 2016b; Algaba et al. 2019). Because of the large KVN beam size leading to larger core blending effect at lower frequencies, the KVN beam does not resolve the core at the lower frequency. Therefore the brightness temperature of the core was underestimated due to the larger source size measured. This effect becomes weaker at higher frequency observations which have relatively smaller beam sizes. In contrast to this, our results show an opposite tendency of brightness temperature to the previous studies using KVN observations, whereas these results are consistent to a previous study on the intrinsic brightness temperature evolution as function of observing frequency using high angular-resolution VLBI observations (Lee 2014). According to Lee (2014), the intrinsic brightness temperature $T_{0}$ of relativistic jets decreases following $T_{0} \propto v_{\text {obs }}^{-1.2}$ with $v_{\mathrm{obs}} \geq 9 \mathrm{GHz}$. In order to check the power index of $T_{B}$, we selected 14 epochs when the core sizes were estimated larger than minimum resolvable sizes at more than three frequencies. We found decreasing trend of core sizes along the observing frequencies in 14 epochs then fitted the $T_{B}$ calculated by these core sizes. We obtained the mean index of $-1.50 \pm 0.21$ (weighted average of -1.11 ), by excluding minimum resolvable sizes, which is a comparable result to that estimated in Lee (2014). Although we are not in a position to directly derive the intrinsic brightness temperature of the relativistic jet using these KVN observations, this result makes us to conclude that the KVN observations of the source are less affected by core blending effect for the $4 \mathrm{C}+29.45$, compared with those of the previous KVN observations for other sources.

For VLBI observations with limited visibility data, we can use a new approach for estimating brightness temperature based on individual visibility measurements and their errors following Lobanov (2015). We can estimate a brightness temperature limit $\left(T_{\mathrm{B}, \mathrm{lim}}\right)$ as described by equation 5 in Lobanov (2015):

$$
\begin{aligned}
T_{\mathrm{B}, \min } & =3.09\left(\frac{B}{\mathrm{~km}}\right)^{2}\left(\frac{V_{q}}{\mathrm{mJy}}\right) \mathrm{K}, \\
T_{\mathrm{B}, \mathrm{lim}} & =1.14\left(\frac{V_{q}+\sigma_{q}}{\mathrm{mJy}}\right)\left(\frac{B}{\mathrm{~km}}\right)^{2}\left(\ln \frac{V_{q}+\sigma_{q}}{V_{q}}\right) \mathrm{K} .
\end{aligned}
$$

We selected KVN observations on MJD 56771, 57037, 57290 and 57503 (closest epochs to the flare 3-6), and estimated the brightness temperature limits at multi frequencies as summarized in Table 7 . We found that the estimated brightness temperature limits $\left(T_{\mathrm{B}, \text { lim }}\right)$ are comparable to those $T_{\mathrm{B}}$ obtained from KVN images, yielding the ratio of $T_{\mathrm{B}, \lim } / T_{\mathrm{B}}=1.82-4.76$. This is consistent to the application results of $T_{\mathrm{B}, \lim } / T_{\mathrm{B}}=4.6$ for the MOJAVE data as reported in Lobanov (2015).

As another way of measuring the brightness temperature of the non-thermal synchrotron emission from the relativistic jets, we investigated the variability brightness temperatures, $T_{\mathrm{B}}^{\mathrm{var}}$, from the obtained variability time scales in Table 2. We found that the mean variability brightness temperature is much higher than the mean brightness temperature derived from the KVN images at $22 \mathrm{GHz}$ and those (Table 7 ) obtained by applying the approach in Lobanov (2015). In order to understand how this difference comes, we calculated the size of the emission region, $\theta_{\mathrm{var}}$, from the variability properties as described in Section 3.5 and compared these $\theta_{\mathrm{var}}$ with the size of the radio core emission regions obtained from the KVN images. The mean core sizes are $0.47 \pm 0.25 \mathrm{mas}, 0.53 \pm 0.22 \mathrm{mas}, 0.42 \pm 0.17 \mathrm{mas}$, and $0.37 \pm 0.34$ mas at $22,43,86$, and $129 \mathrm{GHz}$, respectively. The obtained mean variability size is $0.088 \pm 0.034$ mas derived from the $15 \mathrm{GHz}$ observations, which is smaller than the KVN size at $22 \mathrm{GHz}$ by a factor of 5 . Therefore, we postulate this discrepancy of core sizes may partially affect the difference between brightness temperatures at $22-129 \mathrm{GHz}$ and variability brightness temperatures at $15 \mathrm{GHz}$.

\subsection{Variability Behavior}

We divided the radio light curve into two distinct periods: part A and B (Fig. 2). We attempted to investigate if there is any physical changes in the source over this period.

We cross-compared the flux densities with derived parameters, such as core sizes, turnover frequencies, and Doppler factors, yielding no cross-correlation between the parameters. In particular, as shown in the Tables 2, we did not find any obvious trend in the Doppler factors in section 3.2.1. Similarly, we found no obvious trend in the variability brightness temperatures. This may imply that the strong flaring activities in $4 \mathrm{C}+29.45$ were 
Table 7 Brightness temperatures

\begin{tabular}{cccccccccc}
\hline \hline & $T_{\mathrm{B}, \text { var }}$ & $T_{\mathrm{B}, \lim , 22}$ & $T_{\mathrm{B}, \lim , 43}$ & $T_{\mathrm{B}, \lim , 86}$ & $T_{\mathrm{B}, \lim , 129}$ & $T_{\mathrm{B}, 22}$ & $T_{\mathrm{B}, 43}$ & $T_{\mathrm{B}, 86}$ & $T_{\mathrm{B}, 129}$ \\
MJD & $10^{12} \mathrm{~K}$ & $10^{10} \mathrm{~K}$ & $10^{10} \mathrm{~K}$ & $10^{10} \mathrm{~K}$ & $10^{10} \mathrm{~K}$ & $10^{10} \mathrm{~K}$ & $\begin{array}{c}10^{10} \mathrm{~K} \\
(8)\end{array}$ & $\begin{array}{c}10^{10} \mathrm{~K} \\
(9)\end{array}$ & $\begin{array}{c}10^{10} \mathrm{~K} \\
(10)\end{array}$ \\
\hline 56771 & $192.97 \pm 78.22$ & 3.51 & 2.64 & 1.05 & 0.47 & $>0.25$ & $0.72 \pm 0.04$ & $0.31 \pm 0.02$ & $0.30 \pm 0.01$ \\
57037 & $47.86 \pm 8.60$ & 16.51 & 11.96 & 2.00 & - & $3.73 \pm 0.12$ & $6.56 \pm 0.19$ & $0.42 \pm 0.03$ & - \\
57290 & $23.65 \pm 9.23$ & 11.26 & 2.11 & 1.04 & - & $>1.02$ & $1.08 \pm 0.04$ & $0.25 \pm 0.02$ & - \\
57503 & $41.85 \pm 3.49$ & 8.67 & 10.27 & 3.79 & - & $>1.25$ & $2.19 \pm 0.04$ & $0.95 \pm 0.25$ & - \\
\hline
\end{tabular}

Notes. (1) Epochs closest to the flare 3-6, (2) variability brightness temperatures obtained in Section 3.2.1. (3)-(6) brightness temperature limits at 22, 43, 86 and $129 \mathrm{GHz}$, respectively, following Lobanov(2015), (7)-(10) brightness temperatures obtained from modelfit of KVN data at 22, 43, 86 and $129 \mathrm{GHz}$, respectively.

not attributed to the Doppler factor variability. However, it must be noted that the Doppler factor estimates rely on an intrinsic brightness temperature assumption. It could be that the intrinsic brightness temperature is changing and therefore the Doppler factors as well. We intend to resolve this degeneracy in our future work (Kang et al. in prep.).

We also compared the OVRO data with further multiwavelength data published, including data from the Fermigamma-ray space telescope AGN multi-frequency monitoring alliance (F-GAMMA, Angelakis et al. 2019) and polarimetric monitoring of $\mathrm{AGN}$ at millimeter wavelengths (POLAMI, Agudo et al. 2018) projects and the submillimeter array (SMA) monitoring program. We found two flares (flare 2 and 3) seem to appear in the F-GAMMA (23 and $43 \mathrm{GHz}$ ) and POLAMI (86 and $230 \mathrm{GHz}$ ) light curves. We found three flares (flare 3, 4 and 5) include the flux enhancements appeared in the SMA light curve $(230 \mathrm{GHz})$. For the flare 2, the multi-wavelength flux densities peaked around MJD 56500 at 15, 43, 86 and $230 \mathrm{GHz}$, quasi-simultaneously. The spectrum seems to be inverted between 15 and $43 \mathrm{GHz}$, and steep between 43 and $230 \mathrm{GHz}$. On the other hand, for the flare 3 , the multi-wavelength light curves at 43, 86 and $230 \mathrm{GHz}$ seemed to peak earlier than the OVRO $15 \mathrm{GHz}$ light curve. Moreover the spectrum may appear to be inverted between 15 and $86 \mathrm{GHz}$ and steep between 86 and $230 \mathrm{GHz}$. Although we are not in a position of accurately analysing the multi-wavelengths data at $15-230 \mathrm{GHz}$, the comparison results may imply that during the period of flare 2 and 3 the inverted spectrum (optically thick spectrum) extends to higher frequency (hence higher turnover frequency) and the high energy synchrotron particles increased from the flare 2 to the flare 3 (before the source entered the period B).

\section{Conclusions}

We studied the long-term behavior of a blazar 4C +29.45 which was observed from December 2012 to December 2016. We analyzed KVN data with OVRO $15 \mathrm{GHz}$ data. We found that the OVRO $15 \mathrm{GHz}$ light curve can be divided into two distinguishable periods, period $\mathrm{A}$ and period $\mathrm{B}$, and also found similar variation trends in 22,43 , and $86 \mathrm{GHz}$ light curves. In order to confirm the characteristics of variation, we attempted to estimate the variability time scales using a combined exponential function. Also, we estimated turnover frequencies and maximum flux densities with 22, 43, 86, and $129 \mathrm{GHz}$ KVN data. Then, we estimated $B_{\mathrm{SSA}}$ using turnover frequencies, maximum flux densities, with core sizes from BU data and Doppler factor of $\delta=9.6$ from Jorstad et al. (2017). The $B_{\mathrm{SSA}}$ ranged from 0.001 to $0.099 \mathrm{G}$. In our analysis, we used upper limits for core sizes. In addition, we estimated $B_{\text {eq }}$ assuming the $\eta \sim 100$ that means the jet consist of electrons with both positrons and protons. The $B_{\text {eq }}$ ranged from
0.10 to $0.25 \mathrm{G}$. The $B_{\text {eq }}$ are much greater than the $B_{\mathrm{SSA}}$ for all periods, although we assumed the upper limits and lower limits of $B_{\mathrm{SSA}}$ and $B_{\text {eq }}$, respectively. From the results we concluded that the equipartition region locates upstream the SSA region.

Meanwhile, Fermi-LAT reported an intense flare of gammaray emission from 4C +29.45 in October 2015 (Prince 2019). We will discuss the correlation between gamma-ray flare and behavior of radio emission, such as variation of core sizes and flux density in a follow-up study.

Acknowledgements. We would like to thank the anonymous referee for important comments and suggestions that have enormously improved the manuscript. J.-C. Algaba acknowledges support from the Malaysian Fundamental Research Grant Scheme (FRGS)FRGS/1/2019/STG02/UM/02/6. This work was supported by the National Research Foundation of Korea (NRF) grant funded by the Korea government (MIST) (2020R1A2C2009003). We are grateful to the staff of the KVN who helped to operate the array and to correlate the data. The KVN and a high-performance computing cluster are facilities operated by the KASI (Korea Astronomy and Space Science Institute). The KVN observations and correlations are supported through the high-speed network connections among the KVN sites provided by the KREONET (Korea Research Environment Open NETwork), which is managed and operated by the KISTI (Korea Institute of Science and Technology Information). This research has made use of data from the OVRO 40-m monitoring program (Richards, J. L. et al. 2011, ApJS, 194, 29) which is supported in part by NASA grants NNX08AW31G, NNX11A043G, and NNX14AQ89G and NSF grants AST-0808050 and AST-1109911. This study makes use of $43 \mathrm{GHz}$ VLBA data from the VLBA-BU Blazar Monitoring Program (VLBA-BU-BLAZAR ${ }^{3}$, funded by NASA through the Fermi Guest Investigator Program. The VLBA is an instrument of the National Radio Astronomy Observatory. The National Radio Astronomy Observatory is a facility of the National Science Foundation operated by Associated Universities, Inc. This research has made use of data from the MOJAVE database that is maintained by the MOJAVE team (Lister et al. 2018). J.P. acknowledges financial support from the Korean National Research Foundation (NRF) via Global PhD Fellowship grant 2014H1A2A1018695 and support through the EACOA Fellowship awarded by the East Asia Core Observatories Association, which consists of the Academia Sinica Institute of Astronomy and Astrophysics, the National Astronomical Observatory of Japan, Center for Astronomical Mega-Science, Chinese Academy of Sciences, and the Korea Astronomy and Space Science Institute.

\section{References}

Agudo, I., Thum, C., Ramakrishnan, V., et al. 2018, MNRAS, 473, 1850-1867 Aharonian, F., Akhperjanian, A. G., Bazer-Bachi, A. R., et al. 2007, ApJ, 664:L71-L74

Algaba, J.-C., Gabuzda, D. C., and Smith, P. S., 2012, MNRAS, 420, 542-553

Algaba, J.-C., Zhao, G.-y., Lee, S.-S., et al. 2015, JKAS, 48, 237

Algaba, J.-C., Nakamura, M., Asada, K., et al. 2017, ApJ, 834:65

Algaba, J.-C., Lee, S.-S., Kim, D.-W., et al. 2018a, ApJ, 852:30

Algaba, J.-C., Lee, S.-S., Rani, B., et al. 2018b, ApJ, 859:128

Algaba, J.-C., Hodgson, J., Kang, S., et al. 2019, JKAS, 52: 31-40

Angel, J. \& Stockman, H., 1980, Annu. Rev. Astron. Astrophys, 1980.8:321-61

Angelakis, E., Fuhrmann, L., Myserlis, I., et al. 2019, A\&A, 626, A60

Antonucci, R. 1993, Annu. Rev. Astron. Astrophys, 31:473-521

Asada, K. \& Nakamura, M., 2012, ApJL, 745(2), L28

3 http://www.bu.edu/blazars/VLBAproject.html 
Asada, K., Nakamura, M., Doi, A., Nagai, H., \& Inoue, M., 2014, ApJL, 781(1), L2.

Blandford, R. D., \& Konigl, A., 1978, ApJ, 232: 34-48

Begelman, M. C., Blandford, R. D., \& Rees, M. J., 1984, Rev. Mod. Phys., 56, 255

Edelson, R. A. \& Krolik, J. H., 1993, ApJ, 333:646-659

Event Horizon Telescope Collaboration (Akiyama, K., et al.), 2019, ApJL, 875:L1

Fomalont, E. B. 1999, Synthesis Imaging in Radio Astronomy II, 180, 301

Fuhrmann, L., Krichbaum, T. P., Witzel, A., et al. 2008, A\&A, 490, 1019-1037

Favre, P., Courvoisier, T. J.-L., and Paltani, S., 2005, A\&A 443, 451-463

Fromm, C., M., Perucho, M., Ros, E., 2011, A\&A 531, A95

Gabuzda D. 2017, Galaxies, 5(1), 11

Gurwell, M. A., Peck, A. B., Hostler, S. R., Darrah, M. R., \& Katz, C. A., 2007, in ASP Conf. Ser. 375, From Z-Machines to ALMA: (Sub)millimeter Spectroscopy of Galaxies, ed. A. J. Baker et al. (San Francisco, CA: ASP), 234

Hales, C. A., Beneglia, P., del Palacio, S., et al. 2017, A\&, 598, A42

Hada, K., Kino, M., Doi, A., et al. 2016, ApJ, 817(2), 131

Hodgson, J., Lee, S.-S., Zhao, G.-Y., et al. 2016, JKAS, 49, 137

Hodgson, J., Krichbaum, T. P., Marscher, A. P., et al. 2017, A\&A, 597, 80

Hodgson, J., Rani, B., Lee, S.-S., et al. 2018, MNRAS, 475, 368-378

Hodgson, J., L'Huillier, B., Liodakis, I., et al. 2020, MNRAS, 495, L27-L31

Homan, D. C., Lister, M. L., Kovalev, Y. Y., et al. 2015, ApJ, 798:134

Hovatta, T., Valtaoja, E., Tornikoski, M., and Lähteenmäki, A. 2009, A\&A, 494, 527-537

Jorstad, S., \& Marscher, A., 2016, Galaxies, vol. 4, issue 4, p. 47.

Jorstad, S., Marscher, A., Morozova, D., et al. 2017, ApJ, 846:98

Kataoka, J., \& Stawarz, Ł., 2005, ApJ, 622:797-810

Kim, D.-W., Trippe, S., Lee S.-S., et al. 2017, JKAS, 50, 167-178

Kim, D.-W., Trippe, S., Lee S.-S., et al. 2018, MNRAS, 480, 2324-2333

Kim, J.-Y., Lee, S.-S., Hodgson, J., et al. 2018, A\&A, 610, L5

Kim, J.-Y., Krichbaum, T. P., Lu, R.-S., et al. 2018, A\&A, 616, A188

Komissarov, S. S., Barkov, M. V., Vlahakis, N., \& Königl, A. 2007, MNRAS, 380(1), 51-70.

Kovalev, Y. Y., Lobanov, A. P., Pushkarev, A. B., and Zensus, J. A., 2008, A\&A, 483, 759-768

Lee, S.-S., Lobanov A. P., Krichbaum T. P., et al. 2008, AJ, 136, 159

Lee, S.-S., 2014, JKAS, 47:303-309

Lee, S.-S., Petrov. L., Byun, D.-Y., et al. 2014, AJ, 147:77

Lee, S.-S., Lobanov, A. P., Krichbaum T. P., and Zensus, J. A., 2016, ApJ, 826:135

Lee, S.-S., Wajima, K., Algaba, J.-C., et al. 2016, ApJS, 227, 8

Lee, J. W., Lee, S.-S., Hodgson, J., et al. 2017, ApJ, 841:119

Lee, J. W., Lee, S.-S., Algaba, J.-C., et al. 2020, ApJ, 902:104

Liodakis, I., Hovatta, T., Huppenkothen, D., et al. 2018, ApJ, 866:137

Lister, M. L., Aller, M. F., Aller, H. D., et al. 2018, ApJS, 234:12

Lister, M. L., Homan, D. C., Hovatta, T., et al. 2019, ApJ, 874:43

Liu, B.-R., Liu X., Marchili N., et al. 2013, A\&A, 555, A134

Lobanov, A., P. 1998, A\&A, 330, 79-89

Lobanov, A., P. 2015, A\&A, 574, A84

Madau, P., Ghisellini G., and Persic M., 1987, MNRAS, 224, 257-264

Marscher, A. P., 1983, ApJ, 264, 296

Marscher, A. P., Jorstad, S. G., D’Arcangelo, F. D., et al. 2008, Natur, 452, 966

Martí-Vidal, I., Krichbaum, T. P., Marscher, A. P., et al. 2012, A\&A, 542, A107

Max-Moerbeck, W., Hovatta, T., Richards, J. L., et al. 2014, MNRAS, 445, 428436

Mertens, F., Lobanov, A. P., Walker, R. C., \& Hardee, P. E., 2016, A\&A, 595,A54

Nakamura, M., and Asada, K., 2013, ApJ, 755:118

Nakamura, M., Asada, K., Hada, K., et al. 2018, ApJ, 868(2), 146.

Park, J., Hada, K., Kino, M., et al. 2019a. ApJ, 871(2), 257

Park, J., Lee, S.-S., Kim, J.-Y., et al. 2019b, ApJ, 877:106

Park, J., Hada, K., Kino, M., et al. 2019c, ApJ, 887(2), 147

Peterson, B. M., Wanders, I., Horne, K., et al. 1998, PASP, 110: 660-670

Pilipenko, S. V., Kovalev, Y. Y., Andrianov, A. S., et al. 2018, MNRAS, 474, 3523-3534

Plavin, A. V., Kovalev, Y. Y., Pushkarev, A. B., and Lobanov, A. P., 2019, MNRAS, 485, 1822-1842

Prince, R., Majumdar, P., \& Gupta, N., 2017, ApJ, 844(1), 62.

Prince, R., 2019, ApJ, 871:101

Pushkarev, A. B., Hovatta, T., Kovalev, Y. Y., et al. 2012, A\&A, 545, A113

Raiteri, C. M., Villata, M., D’Ammando, F., 2013, MNRAS, 436, 1530-1545

Rani, B., Krichbaum, T. P., Fuhrmann, L., 2013, A\&A, 552, A11

Richards, J. L., Max-Moerbeck, W., Pavlidou, V., et al. 2011, ApJS, 194, 29

Rybicki, G. B., and Lightman A. P., 1979, Radiative Processes in Astrophysics. Wiley, New York

Valtaoja, E., and Lainela, M., 1999, ApJS, 120:95-99

Véron-Cetty, M. -P., and Véron, P., 2010, A\&A, 518, A10

Walker, R. C., Hardee P. E., Davies, F. B., et al. 2018, ApJ, 855:128

Wang, J.-Y., An, T., Baan W. A., et al. 2014, MNRAS, 443, 58-66

Zhao, G.-Y., Jung, T., Richard, D., et al. 2015, PKAS, 30, 629-631

Zhao, W., Hong X.-Y., An T., et al. 2011, A\&A, 529, A113 


\section{Appendix A: Image and Modelfit Parameters from KVN Observations.}

The image and modelfit parameters obtained from KVN observations, as described in Section 2, are summarized in Table A.1 and A.2

\section{Appendix B: Brightness Temperature}

The definition of brightness temperature is as follows:

$T_{b}=\frac{h v}{k \ln \left(1+\frac{2 h v^{3}}{I_{\nu} c^{2}}\right)}$.

Under the Rayleigh-Jeans approximation, this simplifies to

$T_{b}=\frac{I_{\nu} c^{2}}{2 k v^{2}}$

The intensity $I_{v}$ can be defined as $S / \Omega$, where $\Omega$ is $\pi \theta^{2} / 4 \ln 2$ in circular Gaussian structure. Therefore we can write the equation as

$T_{b}=\frac{c^{2}}{2 k v^{2} \Omega} S=\frac{2 \ln 2}{\pi k} \frac{c^{2} S}{v^{2} \theta^{2}}$.

In order to estimate the brightness temperature assuming that the emission is dominated by variability emission region, we can estimate variability size as

$\theta_{\mathrm{var}}=\frac{c \Delta t}{D_{\mathrm{A}}(1+z)}$

If we replace $\theta$ as $\theta_{\text {var }}$, we can obtain

$T_{b}=\frac{2 \ln 2}{\pi k} \frac{D_{\mathrm{A}}^{2}(1+z)^{2} S}{v^{2} \Delta t^{2}}$.

Here, $v$ is frequency in the rest frame. In order to convert to the observer's frame by referring $v=v_{\mathrm{obs}}(1+z)$ and $D_{\mathrm{A}}=$ $D_{\mathrm{L}} /(1+z)^{2}$, we can obtain

$T_{b}=\frac{2 \ln 2}{\pi k} \frac{D_{\mathrm{L}}^{2} S}{v_{\mathrm{obs}}^{2} \Delta t^{2}(1+z)^{4}}$.

Computing the numerical factor, we obtain a final, convenient expression of

$T_{\mathrm{B}}^{\mathrm{var}}=4.077 \times 10^{13}\left(\frac{D_{\mathrm{L}}}{[\mathrm{Mpc}]}\right)^{2}\left(\frac{v_{\mathrm{obs}}}{[\mathrm{GHz}]}\right)^{-2}\left(\frac{\Delta t}{[\text { day }]}\right)^{-2} \frac{S}{(1+z)^{4}}$.

Table B.1 Computed numerical factors between two different sets of units for brightness temperature estimation.

\begin{tabular}{ccccc}
\hline \hline $\mathrm{S}$ & $D_{\mathrm{L}}$ & $v$ & $\Delta t$ & Numerical factor \\
\hline$[\mathrm{Jy}]$ & {$[\mathrm{m}]$} & {$[\mathrm{Hz}]$} & {$[\mathrm{s}]$} & $3.195 \times 10^{-4}$ \\
{$[\mathrm{Jy}]$} & {$[\mathrm{Mpc}]$} & {$[\mathrm{GHz}]$} & {$[\mathrm{yr}]$} & $3.06 \times 10^{8}$ \\
\hline
\end{tabular}

\section{Appendix C: Error Propagation}

We estimated the uncertainty of magnetic field strength using basic error propagation. When the magnetic field strength $B$ is estimated by $A^{b}$ (i.e. $B \propto A^{b}$ ), the uncertainty, $\sigma_{B}$, can be calculated by $\sigma_{B}=B b \frac{\sigma_{A}}{A}$. Therefore, the uncertainty of magnetic field strength in SSA, $\sigma_{B_{S S A}}$, is proportional to $4 \frac{\sigma_{\theta_{\mathrm{r}}}}{\theta_{\mathrm{r}}}$ and $5 \frac{\sigma_{v_{\mathrm{r}}}}{v_{\mathrm{r}}}$, and the uncertainty of magnetic field strength in equipartition, $\sigma_{B_{\mathrm{eq}}}$, is proportional to $-\frac{6}{7} \frac{\sigma_{\theta_{\mathrm{r}}}}{\theta_{\mathrm{r}}}$ and $\frac{1}{7} \frac{\sigma_{v_{\mathrm{r}}}}{v_{\mathrm{r}}}$. Moreover, the lower uncertainty of $B_{\mathrm{SSA}}$ is larger than $B_{\mathrm{SSA}}$ itself (e.g. $B_{\mathrm{SSA}}<\sigma_{B_{\mathrm{SSA}}}$ ). Therefore we compute the lower uncertainty of $B_{\mathrm{SSA}}$ in logarithm as follows:

$$
\begin{aligned}
\sigma_{f} & =\left|\frac{\sigma_{B_{\mathrm{SSA}}}}{\ln (10) B_{\mathrm{SSA}}}\right| \\
\sigma_{B_{\mathrm{SSA}, \mathrm{low}}} & =B_{\mathrm{SSA}}-10^{-\sigma_{f}} B_{\mathrm{SSA}} .
\end{aligned}
$$


Table A.1. Image Parameters from KVN Observations

\begin{tabular}{|c|c|c|c|c|c|c|c|c|c|c|}
\hline $\begin{array}{l}\text { Date } \\
\text { (1) }\end{array}$ & $\begin{array}{l}\text { MJD } \\
(2)\end{array}$ & $\begin{array}{c}\text { Band } \\
\text { (3) }\end{array}$ & $\begin{array}{c}B_{\max } \\
(\operatorname{mas}) \\
(4)\end{array}$ & $\begin{array}{c}B_{\min } \\
(\mathrm{mas}) \\
(5)\end{array}$ & $\begin{array}{l}B_{\mathrm{pa}} \\
\left({ }^{\circ}\right) \\
(6)\end{array}$ & $\begin{array}{c}S_{\mathrm{KVN}} \\
(\mathrm{Jy}) \\
(7)\end{array}$ & $\begin{array}{c}S_{\mathrm{p}} \\
\text { (Jy/beam) } \\
(8)\end{array}$ & $\begin{array}{c}\sigma_{\mathrm{rms}} \\
(\mathrm{mJy} / \text { beam }) \\
(9)\end{array}$ & $\begin{array}{c}D \\
(10)\end{array}$ & $\begin{array}{c}\xi_{\mathrm{r}} \\
(11)\end{array}$ \\
\hline \multirow[t]{2}{*}{$2012-12-05$} & 56266 & $\mathrm{~K}$ & 6.06 & 3.07 & 72.33 & 0.77 & 0.77 & 6.66 & 115.06 & 0.45 \\
\hline & & Q & 2.93 & 1.68 & 63.5 & 0.65 & 0.64 & 34.31 & 18.63 & 0.48 \\
\hline \multirow[t]{3}{*}{ 2013-01-16 } & 56308 & $\mathrm{~K}$ & 8.61 & 3.08 & -58.75 & 0.79 & 0.78 & 4.77 & 164.23 & 0.40 \\
\hline & & Q & 4.24 & 1.55 & -56.28 & 0.58 & 0.58 & 14.71 & 39.15 & 0.47 \\
\hline & & $\mathrm{D}$ & 2.23 & 0.74 & -55.33 & 0.27 & 0.26 & 13.52 & 19.54 & 0.50 \\
\hline \multirow{4}{*}{ 2013-02-27 } & 56349 & $\mathrm{~K}$ & 6.20 & 2.99 & 76.09 & 0.72 & 0.72 & 10.18 & 70.91 & 0.45 \\
\hline & & Q & 3.27 & 1.43 & 77.16 & 0.70 & 0.70 & 20.88 & 33.29 & 0.47 \\
\hline & & W & 1.65 & 0.71 & 76.09 & 0.54 & 0.52 & 27.81 & 18.87 & 0.47 \\
\hline & & $\mathrm{D}$ & 1.12 & 0.47 & $76.4^{\prime} 4$ & 0.36 & 0.36 & 17.20 & 20.82 & 0.56 \\
\hline \multirow[t]{4}{*}{ 2013-03-28 } & 56381 & $\mathrm{~K}$ & 5.14 & 3.18 & -88.34 & 0.73 & 0.73 & 5.01 & 145.85 & 0.56 \\
\hline & & Q & 2.56 & 1.68 & -82.98 & 0.74 & 0.73 & 15.50 & 47.11 & 0.56 \\
\hline & & $\mathrm{W}$ & 1.30 & 0.76 & 88.19 & 0.59 & 0.56 & 40.39 & 13.94 & 0.58 \\
\hline & & $\mathrm{D}$ & 1.15 & 0.46 & 73.25 & 0.48 & 0.47 & 54.91 & 8.58 & 0.46 \\
\hline \multirow[t]{3}{*}{ 2013-04-11 } & 56394 & $\mathrm{~K}$ & 5.47 & 3.17 & -74.74 & 0.88 & 0.88 & 4.78 & 183.31 & 0.52 \\
\hline & & Q & 2.73 & 1.64 & -73.65 & 0.88 & 0.87 & 13.21 & 66.10 & 0.65 \\
\hline & & $\mathrm{W}$ & 1.34 & 0.77 & -80.12 & 0.78 & 0.74 & 17.63 & 43.98 & 0.57 \\
\hline \multirow[t]{2}{*}{ 2013-05-08 } & 56422 & $\mathrm{~K}$ & 5.37 & 3.31 & -71.12 & 0.87 & 0.87 & 7.62 & 113.77 & 0.48 \\
\hline & & Q & 2.60 & 1.78 & -68.92 & 0.84 & 0.84 & 19.33 & 43.36 & 0.60 \\
\hline \multirow[t]{2}{*}{ 2013-09-24 } & 56559 & $\mathrm{~K}$ & 5.32 & 3.18 & -84.24 & 0.80 & 0.80 & 18.41 & 43.56 & 0.64 \\
\hline & & Q & 3.91 & 1.56 & -65.89 & 0.70 & 0.70 & 7.97 & 87.58 & 0.47 \\
\hline \multirow[t]{3}{*}{ 2013-10-15 } & 56581 & $\mathrm{~K}$ & 5.38 & 3.17 & -83.06 & 0.81 & 0.80 & 9.28 & 86.54 & 0.58 \\
\hline & & Q & 2.79 & 1.57 & -88.83 & 0.74 & 0.74 & 16.57 & 44.51 & 0.51 \\
\hline & & $\mathrm{D}$ & 1.35 & 0.75 & -81.7 & 0.57 & 0.56 & 42.31 & 13.28 & 0.60 \\
\hline \multirow[t]{4}{*}{ 2013-11-20 } & 56616 & $\mathrm{~K}$ & 6.17 & 2.99 & 77.19 & 0.86 & 0.85 & 18.16 & 46.97 & 0.43 \\
\hline & & Q & 3.19 & 1.45 & 75.26 & 0.81 & 0.80 & 18.86 & 42.53 & 0.48 \\
\hline & & $\mathrm{W}$ & 1.64 & 0.71 & 75.51 & 0.73 & 0.72 & 21.47 & 33.32 & 0.49 \\
\hline & & $\mathrm{D}$ & 1.05 & 0.50 & 79.88 & 0.58 & 0.57 & 20.96 & 27.34 & 0.54 \\
\hline \multirow[t]{4}{*}{ 2013-12-24 } & 56651 & $\mathrm{~K}$ & 6.62 & 2.91 & -61.13 & 0.81 & 0.80 & 5.79 & 138.97 & 0.47 \\
\hline & & Q & 3.16 & 1.53 & -60.41 & 0.72 & 0.71 & 10.66 & 66.64 & 0.47 \\
\hline & & $\mathrm{W}$ & 1.86 & 0.66 & -62.15 & 0.53 & 0.50 & 29.23 & 17.10 & 0.48 \\
\hline & & $\mathrm{D}$ & 1.28 & 0.44 & -61.87 & 0.34 & 0.33 & 21.98 & 15.11 & 0.45 \\
\hline \multirow[t]{3}{*}{ 2014-02-28 } & 56716 & $\mathrm{~K}$ & 6.30 & 3.15 & -64.03 & 0.81 & 0.81 & 5.05 & 159.42 & 0.59 \\
\hline & & Q & 2.80 & 1.77 & -76.63 & 0.74 & 0.74 & 5.20 & 141.52 & 0.60 \\
\hline & & $\mathrm{W}$ & 1.80 & 0.71 & -61.59 & 0.56 & 0.55 & 19.06 & 28.89 & 0.58 \\
\hline \multirow[t]{4}{*}{ 2014-03-22 } & 56740 & $\mathrm{~K}$ & 5.37 & 2.99 & -75.45 & 0.85 & 0.85 & 4.98 & 170.71 & 0.58 \\
\hline & & Q & 2.62 & 1.56 & -74.61 & 0.80 & 0.79 & 10.86 & 73.15 & 0.72 \\
\hline & & $\mathrm{W}$ & 1.40 & 0.71 & -76.79 & 0.61 & 0.58 & 23.84 & 24.42 & 0.70 \\
\hline & & $\mathrm{D}$ & 0.96 & 0.48 & -72.7 & 0.32 & 0.31 & 21.50 & 14.49 & 0.71 \\
\hline \multirow[t]{4}{*}{ 2014-04-23 } & 56771 & $\mathrm{~K}$ & 5.31 & 3.03 & -78.69 & 0.86 & 0.86 & 4.77 & 180.07 & 0.64 \\
\hline & & Q & 2.63 & 1.54 & -77.77 & 0.90 & 0.90 & 7.09 & 126.72 & 0.80 \\
\hline & & $\mathrm{W}$ & 1.40 & 0.72 & -80.88 & 0.79 & 0.82 & 16.60 & 49.40 & 0.81 \\
\hline & & $\mathrm{D}$ & 0.97 & 0.48 & -74.73 & 0.58 & 0.57 & 22.06 & 25.86 & 0.79 \\
\hline \multirow[t]{4}{*}{ 2014-09-02 } & 56903 & $\mathrm{~K}$ & 5.23 & 3.04 & -82.95 & 1.08 & 1.08 & 2.27 & 474.39 & 0.44 \\
\hline & & $\mathrm{Q}$ & 2.59 & 1.53 & -79.77 & 1.12 & 1.12 & 9.39 & 119.12 & 0.44 \\
\hline & & $\mathrm{W}$ & 1.37 & 0.74 & -87.21 & 0.88 & 0.87 & 15.37 & 56.58 & 0.56 \\
\hline & & $\mathrm{D}$ & 1.36 & 0.74 & -85.89 & 0.92 & 0.91 & 40.35 & 22.66 & 0.51 \\
\hline 2014-09-27 & 56928 & $\mathrm{~K}$ & 5.33 & 2.98 & -80.54 & 1.04 & 1.03 & 11.60 & 89.12 & 0.67 \\
\hline & & $\mathrm{W}$ & 1.53 & 0.72 & -60.79 & 0.80 & 0.78 & 35.51 & 21.87 & 0.51 \\
\hline 2014-10-29 & 56960 & $\mathrm{~K}$ & 5.67 & 2.81 & -87.99 & 1.21 & 1.20 & 8.96 & 134.15 & 0.63 \\
\hline & & Q & 2.86 & 1.40 & -88.22 & 1.08 & 1.07 & 13.17 & 81.35 & 0.69 \\
\hline & & $\mathrm{W}$ & 1.47 & 0.68 & -87.72 & 0.75 & 0.73 & 19.07 & 38.19 & 0.62 \\
\hline $2014-11-28$ & 56989 & $\mathrm{~K}$ & 5.71 & 2.82 & -75.66 & 1.54 & 1.53 & 21.04 & 72.54 & 0.73 \\
\hline & & $\mathrm{Q}$ & 2.79 & 1.46 & -71.32 & 1.58 & 1.57 & 9.41 & 167.27 & 0.64 \\
\hline & & $\mathrm{W}$ & 1.48 & 0.70 & -73.56 & 1.08 & 1.13 & 38.51 & 29.39 & 0.59 \\
\hline $2014-12-26$ & 57018 & $\mathrm{~K}$ & 5.54 & 2.88 & -82.83 & 2.02 & 2.02 & 10.70 & 188.90 & 0.66 \\
\hline $2015-01-15$ & 57037 & $\mathrm{~K}$ & 5.37 & 2.93 & -83.47 & 2.11 & 2.09 & 17.16 & 122.04 & 0.72 \\
\hline & & Q & 2.57 & 1.57 & -79.39 & 1.93 & 1.93 & 14.65 & 131.38 & 0.75 \\
\hline & & $\mathrm{W}$ & 1.38 & 0.71 & -81.48 & 1.38 & 1.36 & 36.28 & 37.51 & 0.93 \\
\hline
\end{tabular}


Table A.1. continued.

\begin{tabular}{|c|c|c|c|c|c|c|c|c|c|c|}
\hline $\begin{array}{l}\text { Date } \\
\text { (1) }\end{array}$ & $\begin{array}{l}\text { MJD } \\
(2)\end{array}$ & $\begin{array}{c}\text { Band } \\
\text { (3) }\end{array}$ & $\begin{array}{c}B_{\max } \\
(\operatorname{mas}) \\
(4)\end{array}$ & $\begin{array}{c}B_{\min } \\
(\mathrm{mas}) \\
(5)\end{array}$ & $\begin{array}{l}B_{\mathrm{pa}} \\
\left({ }^{\circ}\right) \\
(6)\end{array}$ & $\begin{array}{c}S_{\text {KVN }} \\
(\mathrm{Jy}) \\
(7)\end{array}$ & $\begin{array}{c}S_{\mathrm{p}} \\
\text { (Jy/beam) } \\
(8)\end{array}$ & $\begin{array}{c}\sigma_{\mathrm{rms}} \\
(\mathrm{mJy} / \text { beam }) \\
(9)\end{array}$ & $\begin{array}{c}D \\
(10)\end{array}$ & $\begin{array}{c}\xi_{\mathrm{r}} \\
(11)\end{array}$ \\
\hline \multirow[t]{3}{*}{$2015-02-23$} & 57076 & $\mathrm{~K}$ & 5.37 & 2.95 & -86.16 & 2.59 & 2.59 & 8.18 & 317.11 & 0.51 \\
\hline & & Q & 2.60 & 1.54 & -87.84 & 2.47 & 2.47 & 11.54 & 213.79 & 0.56 \\
\hline & & $\mathrm{W}$ & 1.39 & 0.71 & -85.68 & 1.94 & 1.92 & 32.87 & 58.36 & 0.79 \\
\hline \multirow[t]{3}{*}{$2015-03-26$} & 57109 & $\mathrm{~K}$ & 5.46 & 2.93 & -79.81 & 2.43 & 2.43 & 6.81 & 356.55 & 0.63 \\
\hline & & Q & 2.63 & 1.53 & -78.66 & 2.09 & 2.08 & 17.41 & 119.38 & 0.63 \\
\hline & & $\mathrm{W}$ & 1.39 & 0.72 & -81.74 & 1.38 & 1.34 & 33.44 & 40.03 & 0.70 \\
\hline \multirow[t]{3}{*}{ 2015-04-30 } & 57144 & $\mathrm{~K}$ & 6.47 & 2.67 & 79.66 & 1.94 & 1.94 & 25.24 & 77.01 & 0.61 \\
\hline & & W & 1.77 & 0.63 & 79.99 & 1.21 & 1.20 & 39.64 & 30.39 & 0.68 \\
\hline & & $\mathrm{D}$ & 1.10 & 0.44 & 88.57 & 1.13 & 1.10 & 125.81 & 8.76 & 0.48 \\
\hline \multirow{3}{*}{ 2015-09-24 } & 57290 & $\mathrm{~K}$ & 5.61 & 2.95 & -70.86 & 1.92 & 1.92 & 16.46 & 116.62 & 0.90 \\
\hline & & Q & 3.04 & 1.45 & -68.40 & 1.66 & 1.65 & 12.37 & 133.21 & 0.68 \\
\hline & & $\mathrm{W}$ & 1.57 & 0.69 & -70.09 & 1.19 & 1.16 & 21.52 & 53.71 & 0.64 \\
\hline \multirow[t]{3}{*}{$2015-10-23$} & 57319 & $\mathrm{~K}$ & 5.37 & 2.97 & -88.90 & 1.79 & 1.79 & 14.14 & 126.63 & 0.84 \\
\hline & & Q & 2.85 & 1.43 & -87.98 & 1.45 & 1.44 & 13.09 & 110.31 & 0.75 \\
\hline & & $\mathrm{W}$ & 1.50 & 0.69 & 86.09 & 1.03 & 1.01 & 38.81 & 25.98 & 0.66 \\
\hline \multirow[t]{4}{*}{ 2015-11-30 } & 57357 & $\mathrm{~K}$ & 5.17 & 3.13 & -85.41 & 1.79 & 1.79 & 11.53 & 155.14 & 0.87 \\
\hline & & $\mathrm{Q}$ & 2.59 & 1.56 & -84.21 & 1.68 & 1.68 & 9.74 & 172.09 & 0.63 \\
\hline & & $\mathrm{W}$ & 1.33 & 0.76 & -86.73 & 1.33 & 1.32 & 20.92 & 63.07 & 0.82 \\
\hline & & $\mathrm{D}$ & 0.88 & 0.52 & -83.01 & 0.87 & 0.87 & 41.42 & 20.97 & 0.75 \\
\hline \multirow[t]{4}{*}{$2015-12-28$} & 57385 & $\mathrm{~K}$ & 5.31 & 3.08 & -85.92 & 1.81 & 1.81 & 13.77 & 131.24 & 0.95 \\
\hline & & Q & 2.65 & 1.56 & -86.71 & 1.82 & 1.81 & 7.39 & 245.50 & 0.80 \\
\hline & & $\mathrm{W}$ & 1.40 & 0.72 & -86.49 & 1.38 & 1.36 & 15.61 & 86.94 & 0.76 \\
\hline & & $\mathrm{D}$ & 0.91 & 0.45 & -81.19 & 0.72 & 0.72 & 11.33 & 63.41 & 0.81 \\
\hline \multirow[t]{4}{*}{ 2016-01-13 } & 57401 & $\mathrm{~K}$ & 5.26 & 3.11 & -88.32 & 1.69 & 1.69 & 9.20 & 183.72 & 0.69 \\
\hline & & Q & 2.66 & 1.53 & 88.50 & 1.77 & 1.76 & 9.00 & 196.54 & 0.64 \\
\hline & & $\mathrm{W}$ & 1.40 & 0.73 & -89.84 & 1.55 & 1.52 & 23.02 & 67.22 & 0.78 \\
\hline & & D & 0.97 & 0.48 & 86.90 & 1.04 & 1.03 & 26.51 & 39.18 & 0.73 \\
\hline \multirow[t]{4}{*}{ 2016-03-01 } & 57449 & $\mathrm{~K}$ & 5.41 & 3.00 & -80.80 & 2.39 & 2.39 & 11.74 & 203.32 & 0.80 \\
\hline & & Q & 2.74 & 1.48 & -79.05 & 2.42 & 2.40 & 17.66 & 135.99 & 0.83 \\
\hline & & W & 1.38 & 0.73 & -81.56 & 1.84 & 1.82 & 22.69 & 80.23 & 0.84 \\
\hline & & $\mathrm{D}$ & 0.91 & 0.52 & -73.93 & 1.37 & 1.34 & 28.27 & 48.53 & 0.76 \\
\hline \multirow[t]{3}{*}{ 2016-04-24 } & 57503 & $\mathrm{~K}$ & 5.13 & 3.12 & -80.46 & 2.88 & 2.88 & 49.79 & 57.89 & 0.76 \\
\hline & & Q & 2.63 & 1.53 & -80.71 & 2.80 & 2.76 & 17.99 & 155.59 & 0.61 \\
\hline & & $\mathrm{W}$ & 1.33 & 0.76 & -85.64 & 1.89 & 1.87 & 27.17 & 68.82 & 0.75 \\
\hline \multirow[t]{2}{*}{$2016-08-23$} & 57624 & $\mathrm{~K}$ & 6.44 & 3.05 & -57.94 & 1.38 & 1.38 & 16.30 & 84.83 & 0.54 \\
\hline & & $\mathrm{D}$ & 1.06 & 0.49 & -63.06 & 0.78 & 0.77 & 69.99 & 10.93 & 0.46 \\
\hline \multirow[t]{4}{*}{$2016-10-18$} & 57680 & $\mathrm{~K}$ & 5.38 & 2.96 & -88.14 & 1.18 & 1.18 & 10.56 & 112.16 & 0.79 \\
\hline & & Q & 2.92 & 1.37 & -83.95 & 1.08 & 1.08 & 11.50 & 93.68 & 0.74 \\
\hline & & $\mathrm{W}$ & 1.39 & 0.72 & 88.08 & 0.87 & 0.87 & 19.39 & 44.82 & 0.75 \\
\hline & & D & 0.90 & 0.52 & 81.26 & 0.68 & 0.66 & 43.65 & 15.16 & 0.70 \\
\hline \multirow[t]{3}{*}{ 2016-11-27 } & 57719 & $\mathrm{~K}$ & 5.22 & 3.35 & 87.10 & 1.49 & 1.49 & 9.62 & 154.60 & 0.80 \\
\hline & & $\mathrm{Q}$ & 3.11 & 1.51 & 71.55 & 1.24 & 1.23 & 37.08 & 33.24 & 0.45 \\
\hline & & $\mathrm{W}$ & 1.56 & 0.77 & 68.18 & 1.14 & 1.13 & 29.35 & 38.39 & 0.48 \\
\hline \multirow[t]{4}{*}{$2016-12-28$} & 57750 & $\mathrm{~K}$ & 5.12 & 3.14 & -85.72 & 1.95 & 1.95 & 12.93 & 150.86 & 0.75 \\
\hline & & Q & 2.68 & 1.50 & -88.74 & 1.71 & 1.70 & 20.09 & 84.69 & 0.84 \\
\hline & & $\mathrm{W}$ & 1.29 & 0.78 & -87.96 & 1.25 & 1.24 & 32.24 & 38.51 & 0.74 \\
\hline & & D & 0.81 & 0.59 & -86.55 & 0.79 & 0.79 & 31.10 & 25.36 & 0.74 \\
\hline
\end{tabular}

Notes. (1) Date in Year-Month-Date, (2) Modified Julian Date, (3) observing frequency band, (4) major axis of beam, (5) minor axis of beam, (6) position angle of major axis, (7) CLEANed total flux density, (8) CLEANed peak flux density, (9) rms noise, (10) dynamic range, (11) image quality. 
Table A.2. MODELFIT parameters from KVN Observations.

\begin{tabular}{|c|c|c|c|c|c|c|}
\hline $\begin{array}{l}\text { Date } \\
\text { (1) }\end{array}$ & $\begin{array}{c}\text { MJD } \\
(2)\end{array}$ & $\begin{array}{c}\text { Band } \\
\text { (3) }\end{array}$ & $\begin{array}{l}S_{\text {total }} \\
\text { (Jy) } \\
(4)\end{array}$ & $\begin{array}{c}S_{\text {peak }} \\
\text { (Jy/beam) } \\
(5)\end{array}$ & $\begin{array}{c}d \\
\text { (mas) } \\
(6)\end{array}$ & $\begin{array}{c}T_{\mathrm{b}} \\
\left(10^{10} \mathrm{~K}\right) \\
(7)\end{array}$ \\
\hline \multirow[t]{2}{*}{$2012-12-05$} & 56266 & $\mathrm{~K}$ & $0.78 \pm 0.01$ & $0.76 \pm 0.01$ & $0.62 \pm 0.01$ & $0.93 \pm 0.03$ \\
\hline & & Q & $0.82 \pm 0.03$ & $0.64 \pm 0.02$ & $1.12 \pm 0.08$ & $0.07 \pm 0.01$ \\
\hline \multirow[t]{3}{*}{ 2013-01-16 } & 56308 & $\mathrm{~K}$ & $0.81 \pm 0.00$ & $0.78 \pm 0.00$ & $0.75 \pm 0.00$ & $0.65 \pm 0.01$ \\
\hline & & $\mathrm{W}$ & $0.37 \pm 0.02$ & $0.25 \pm 0.01$ & $0.72 \pm 0.07$ & $0.02 \pm 0.00$ \\
\hline & & $\mathrm{D}$ & $0.33 \pm 0.00$ & $0.27 \pm 0.00$ & $0.51 \pm 0.03$ & $0.02 \pm 0.00$ \\
\hline \multirow[t]{4}{*}{ 2013-02-27 } & 56349 & $\mathrm{~K}$ & $0.76 \pm 0.01$ & $0.72 \pm 0.01$ & $0.93 \pm 0.02$ & $0.39 \pm 0.02$ \\
\hline & & Q & $0.80 \pm 0.02$ & $0.69 \pm 0.01$ & $0.76 \pm 0.03$ & $0.16 \pm 0.01$ \\
\hline & & $\mathrm{W}$ & $0.68 \pm 0.03$ & $0.53 \pm 0.02$ & $0.52 \pm 0.03$ & $0.07 \pm 0.01$ \\
\hline & & $\mathrm{D}$ & $0.32 \pm 0.05$ & $0.35 \pm 0.04$ & $0.23 \pm 0.11$ & $0.08 \pm 0.09$ \\
\hline \multirow[t]{4}{*}{ 2013-03-28 } & 56381 & $\mathrm{~K}$ & $0.76 \pm 0.01$ & $0.73 \pm 0.00$ & $0.80 \pm 0.01$ & $0.53 \pm 0.01$ \\
\hline & & $\mathrm{Q}$ & $0.82 \pm 0.02$ & $0.72 \pm 0.01$ & $0.72 \pm 0.03$ & $0.18 \pm 0.02$ \\
\hline & & $\mathrm{W}$ & $0.86 \pm 0.04$ & $0.58 \pm 0.02$ & $0.67 \pm 0.06$ & $0.06 \pm 0.01$ \\
\hline & & $\mathrm{D}$ & $1.07 \pm 0.07$ & $0.57 \pm 0.03$ & $0.62 \pm 0.12$ & $0.04 \pm 0.02$ \\
\hline \multirow[t]{3}{*}{ 2013-04-11 } & 56394 & $\mathrm{~K}$ & $0.89 \pm 0.01$ & $0.87 \pm 0.01$ & $0.51 \pm 0.00$ & $1.51 \pm 0.03$ \\
\hline & & Q & $0.92 \pm 0.02$ & $0.87 \pm 0.01$ & $0.50 \pm 0.01$ & $0.41 \pm 0.02$ \\
\hline & & $\mathrm{W}$ & $0.95 \pm 0.07$ & $0.59 \pm 0.04$ & $0.40 \pm 0.01$ & $0.15 \pm 0.01$ \\
\hline \multirow{2}{*}{ 2013-05-08 } & 56422 & $\mathrm{~K}$ & $0.91 \pm 0.01$ & $0.86 \pm 0.01$ & $0.94 \pm 0.02$ & $0.47 \pm 0.02$ \\
\hline & & W & $0.84 \pm 0.08$ & $0.49 \pm 0.04$ & $0.80 \pm 0.15$ & $0.04 \pm 0.02$ \\
\hline \multirow[t]{2}{*}{ 2013-09-24 } & 56559 & $\mathrm{~K}$ & $0.83 \pm 0.03$ & $0.80 \pm 0.02$ & $0.70 \pm 0.01$ & $0.75 \pm 0.03$ \\
\hline & & Q & $0.71 \pm 0.01$ & $0.70 \pm 0.01$ & $0.33 \pm 0.04$ & $0.72 \pm 0.02$ \\
\hline \multirow[t]{3}{*}{ 2013-10-15 } & 56581 & $\mathrm{~K}$ & $0.80 \pm 0.01$ & $0.80 \pm 0.01$ & $<1.15$ & $>0.27$ \\
\hline & & Q & $0.75 \pm 0.02$ & $0.74 \pm 0.02$ & $0.30 \pm 0.00$ & $0.94 \pm 0.03$ \\
\hline & & $\mathrm{D}$ & $0.78 \pm 0.06$ & $0.57 \pm 0.03$ & $0.59 \pm 0.06$ & $0.03 \pm 0.01$ \\
\hline \multirow[t]{4}{*}{ 2013-11-20 } & 56616 & $\mathrm{~K}$ & $0.87 \pm 0.03$ & $0.85 \pm 0.02$ & $0.52 \pm 0.01$ & $1.42 \pm 0.05$ \\
\hline & & $\mathrm{Q}$ & $0.88 \pm 0.02$ & $0.80 \pm 0.01$ & $0.61 \pm 0.02$ & $0.26 \pm 0.02$ \\
\hline & & $\mathrm{W}$ & $0.85 \pm 0.01$ & $0.71 \pm 0.01$ & $0.41 \pm 0.01$ & $0.14 \pm 0.01$ \\
\hline & & $\mathrm{D}$ & $0.66 \pm 0.03$ & $0.57 \pm 0.02$ & $0.25 \pm 0.01$ & $0.13 \pm 0.02$ \\
\hline \multirow[t]{4}{*}{$2013-12-24$} & 56651 & $\mathrm{~K}$ & $0.83 \pm 0.01$ & $0.80 \pm 0.01$ & $0.67 \pm 0.01$ & $0.83 \pm 0.02$ \\
\hline & & $\mathrm{Q}$ & $0.77 \pm 0.01$ & $0.71 \pm 0.01$ & $0.58 \pm 0.01$ & $0.25 \pm 0.01$ \\
\hline & & $\mathrm{W}$ & $0.70 \pm 0.02$ & $0.51 \pm 0.01$ & $0.56 \pm 0.03$ & $0.06 \pm 0.01$ \\
\hline & & $\mathrm{D}$ & $0.44 \pm 0.03$ & $0.34 \pm 0.02$ & $0.33 \pm 0.01$ & $0.05 \pm 0.01$ \\
\hline \multirow[t]{3}{*}{ 2014-02-28 } & 56716 & $\mathrm{~K}$ & $0.80 \pm 0.01$ & $0.81 \pm 0.01$ & $<1.37$ & $>0.19$ \\
\hline & & Q & $0.76 \pm 0.01$ & $0.73 \pm 0.01$ & $0.40 \pm 0.00$ & $0.54 \pm 0.01$ \\
\hline & & $\mathrm{W}$ & $0.61 \pm 0.03$ & $0.55 \pm 0.02$ & $0.33 \pm 0.01$ & $0.16 \pm 0.01$ \\
\hline \multirow{4}{*}{ 2014-03-22 } & 56740 & $\mathrm{~K}$ & $0.86 \pm 0.01$ & $0.85 \pm 0.01$ & $0.38 \pm 0.00$ & $2.64 \pm 0.05$ \\
\hline & & $\mathrm{Q}$ & $0.88 \pm 0.02$ & $0.79 \pm 0.01$ & $0.64 \pm 0.02$ & $0.24 \pm 0.02$ \\
\hline & & $\mathrm{W}$ & $0.81 \pm 0.03$ & $0.59 \pm 0.02$ & $0.56 \pm 0.03$ & $0.07 \pm 0.01$ \\
\hline & & $\mathrm{D}$ & $0.35 \pm 0.03$ & $0.31 \pm 0.02$ & $0.23 \pm 0.02$ & $0.09 \pm 0.01$ \\
\hline \multirow[t]{4}{*}{ 2014-04-23 } & 56771 & $\mathrm{~K}$ & $0.85 \pm 0.01$ & $0.86 \pm 0.01$ & $<1.23$ & $>0.25$ \\
\hline & & Q & $0.93 \pm 0.01$ & $0.90 \pm 0.01$ & $0.38 \pm 0.01$ & $0.72 \pm 0.04$ \\
\hline & & $\mathrm{W}$ & $0.89 \pm 0.02$ & $0.82 \pm 0.02$ & $0.28 \pm 0.01$ & $0.31 \pm 0.02$ \\
\hline & & $\mathrm{D}$ & $0.61 \pm 0.03$ & $0.57 \pm 0.02$ & $0.16 \pm 0.00$ & $0.30 \pm 0.01$ \\
\hline \multirow[t]{4}{*}{ 2014-09-02 } & 56903 & $\mathrm{~K}$ & $1.07 \pm 0.01$ & $1.08 \pm 0.01$ & $<1.37$ & $>0.26$ \\
\hline & & Q & $1.11 \pm 0.02$ & $1.12 \pm 0.01$ & $<0.33$ & $>1.18$ \\
\hline & & $\mathrm{W}$ & $0.98 \pm 0.01$ & $0.86 \pm 0.01$ & $0.34 \pm 0.00$ & $0.24 \pm 0.01$ \\
\hline & & $\mathrm{D}$ & $1.20 \pm 0.01$ & $0.92 \pm 0.01$ & $0.52 \pm 0.01$ & $0.06 \pm 0.00$ \\
\hline \multirow[t]{2}{*}{ 2014-09-27 } & 56928 & $\mathrm{~K}$ & $1.08 \pm 0.02$ & $1.03 \pm 0.01$ & $0.79 \pm 0.01$ & $0.77 \pm 0.01$ \\
\hline & & $\mathrm{W}$ & $0.90 \pm 0.06$ & $0.77 \pm 0.04$ & $0.38 \pm 0.03$ & $0.17 \pm 0.03$ \\
\hline \multirow[t]{3}{*}{ 2014-10-29 } & 56960 & $\mathrm{~K}$ & $1.27 \pm 0.01$ & $1.20 \pm 0.01$ & $0.88 \pm 0.01$ & $0.74 \pm 0.02$ \\
\hline & & Q & $1.13 \pm 0.02$ & $1.07 \pm 0.01$ & $0.42 \pm 0.01$ & $0.70 \pm 0.05$ \\
\hline & & $\mathrm{W}$ & $0.84 \pm 0.03$ & $0.72 \pm 0.02$ & $0.35 \pm 0.01$ & $0.19 \pm 0.02$ \\
\hline $2014-11-28$ & 56989 & $\mathrm{~K}$ & $1.65 \pm 0.03$ & $1.52 \pm 0.02$ & $1.07 \pm 0.04$ & $0.64 \pm 0.05$ \\
\hline & & Q & $1.59 \pm 0.01$ & $1.57 \pm 0.01$ & $0.20 \pm 0.00$ & $4.35 \pm 0.09$ \\
\hline & & $\mathrm{W}$ & $1.35 \pm 0.04$ & $1.13 \pm 0.03$ & $0.40 \pm 0.04$ & $0.24 \pm 0.05$ \\
\hline $2014-12-26$ & 57018 & $\mathrm{~K}$ & $2.03 \pm 0.02$ & $2.02 \pm 0.01$ & $0.26 \pm 0.00$ & $13.17 \pm 0.14$ \\
\hline $2015-01-15$ & 57037 & $\mathrm{~K}$ & $2.13 \pm 0.03$ & $2.09 \pm 0.02$ & $0.51 \pm 0.01$ & $3.73 \pm 0.12$ \\
\hline & & $\mathrm{Q}$ & $1.94 \pm 0.02$ & $1.92 \pm 0.02$ & $0.18 \pm 0.00$ & $6.56 \pm 0.19$ \\
\hline & & $\mathrm{W}$ & $1.52 \pm 0.05$ & $1.35 \pm 0.04$ & $0.32 \pm 0.01$ & $0.42 \pm 0.03$ \\
\hline
\end{tabular}


Table A.2. continued.

\begin{tabular}{|c|c|c|c|c|c|c|}
\hline $\begin{array}{l}\text { Date } \\
(1)\end{array}$ & $\begin{array}{l}\text { MJD } \\
(2)\end{array}$ & $\begin{array}{c}\text { Band } \\
\text { (3) }\end{array}$ & $\begin{array}{c}S_{\text {total }} \\
(\mathrm{Jy}) \\
(4)\end{array}$ & $\begin{array}{c}S_{\text {peak }} \\
\text { (Jy/beam) } \\
(5)\end{array}$ & $\begin{array}{c}d \\
(\mathrm{mas}) \\
(6)\end{array}$ & $\begin{array}{c}T_{\mathrm{b}} \\
\left(10^{10} \mathrm{~K}\right) \\
(7)\end{array}$ \\
\hline \multirow[t]{3}{*}{$2015-02-23$} & 57076 & $\mathrm{~K}$ & $2.58 \pm 0.01$ & $2.59 \pm 0.01$ & $<1.10$ & $>0.96$ \\
\hline & & Q & $2.54 \pm 0.01$ & $2.46 \pm 0.01$ & $0.35 \pm 0.00$ & $2.36 \pm 0.03$ \\
\hline & & $\mathrm{W}$ & $2.03 \pm 0.05$ & $1.91 \pm 0.03$ & $0.23 \pm 0.01$ & $1.07 \pm 0.07$ \\
\hline \multirow[t]{3}{*}{$2015-03-26$} & 57109 & $\mathrm{~K}$ & $2.43 \pm 0.01$ & $2.43 \pm 0.01$ & $<0.21$ & $>26.04$ \\
\hline & & $\mathrm{Q}$ & $2.20 \pm 0.02$ & $2.07 \pm 0.02$ & $0.48 \pm 0.01$ & $1.06 \pm 0.04$ \\
\hline & & $\mathrm{W}$ & $1.68 \pm 0.04$ & $1.34 \pm 0.03$ & $0.46 \pm 0.04$ & $0.23 \pm 0.05$ \\
\hline \multirow[t]{3}{*}{ 2015-04-30 } & 57144 & $\mathrm{~K}$ & $1.94 \pm 0.04$ & $1.94 \pm 0.03$ & $<1.27$ & $>0.54$ \\
\hline & & W & $1.20 \pm 0.06$ & $1.20 \pm 0.04$ & $<0.07$ & $>7.27$ \\
\hline & & $\mathrm{D}$ & $2.15 \pm 0.12$ & $1.26 \pm 0.06$ & $0.52 \pm 0.04$ & $0.10 \pm 0.02$ \\
\hline \multirow[t]{3}{*}{ 2015-09-24 } & 57290 & $\mathrm{~K}$ & $1.92 \pm 0.02$ & $1.92 \pm 0.02$ & $<0.92$ & $>1.02$ \\
\hline & & Q & $1.73 \pm 0.02$ & $1.64 \pm 0.01$ & $0.42 \pm 0.01$ & $1.08 \pm 0.04$ \\
\hline & & $\mathrm{W}$ & $1.36 \pm 0.03$ & $1.15 \pm 0.02$ & $0.39 \pm 0.01$ & $0.25 \pm 0.02$ \\
\hline \multirow[t]{3}{*}{$2015-10-23$} & 57319 & $\mathrm{~K}$ & $1.78 \pm 0.02$ & $1.79 \pm 0.02$ & $<1.17$ & $>0.58$ \\
\hline & & Q & $1.53 \pm 0.02$ & $1.44 \pm 0.01$ & $0.45 \pm 0.01$ & $0.84 \pm 0.05$ \\
\hline & & W & $1.30 \pm 0.05$ & $1.01 \pm 0.03$ & $0.46 \pm 0.06$ & $0.17 \pm 0.05$ \\
\hline \multirow[t]{4}{*}{$2015-11-30$} & 57357 & $\mathrm{~K}$ & $1.78 \pm 0.02$ & $1.79 \pm 0.01$ & $<1.22$ & $>0.54$ \\
\hline & & Q & $1.74 \pm 0.01$ & $1.67 \pm 0.01$ & $0.38 \pm 0.00$ & $1.37 \pm 0.03$ \\
\hline & & $\mathrm{W}$ & $1.59 \pm 0.04$ & $1.31 \pm 0.02$ & $0.39 \pm 0.08$ & $0.30 \pm 0.15$ \\
\hline & & $\mathrm{D}$ & $0.84 \pm 0.06$ & $0.86 \pm 0.04$ & $0.12 \pm 0.03$ & $0.72 \pm 0.45$ \\
\hline \multirow[t]{4}{*}{$2015-12-28$} & 57385 & $\mathrm{~K}$ & $1.83 \pm 0.02$ & $1.80 \pm 0.02$ & $<0.98$ & $>0.86$ \\
\hline & & $\mathrm{Q}$ & $1.89 \pm 0.01$ & $1.81 \pm 0.01$ & $0.41 \pm 0.01$ & $1.26 \pm 0.04$ \\
\hline & & $\mathrm{W}$ & $1.49 \pm 0.03$ & $1.35 \pm 0.02$ & $<0.21$ & $>0.93$ \\
\hline & & D & $0.74 \pm 0.02$ & $0.72 \pm 0.01$ & $0.11 \pm 0.00$ & $0.85 \pm 0.01$ \\
\hline \multirow[t]{4}{*}{ 2016-01-13 } & 57401 & $\mathrm{~K}$ & $1.68 \pm 0.02$ & $1.69 \pm 0.01$ & $<1.39$ & $>0.39$ \\
\hline & & Q & $1.89 \pm 0.03$ & $1.55 \pm 0.02$ & $<0.28$ & $>2.57$ \\
\hline & & W & $1.90 \pm 0.07$ & $1.21 \pm 0.04$ & $<0.22$ & $>0.86$ \\
\hline & & $\mathrm{D}$ & $1.23 \pm 0.07$ & $0.79 \pm 0.04$ & $0.04 \pm 0.00$ & $8.77 \pm 1.67$ \\
\hline \multirow[t]{4}{*}{ 2016-03-01 } & 57449 & $\mathrm{~K}$ & $2.35 \pm 0.02$ & $2.38 \pm 0.02$ & $<1.61$ & $>0.40$ \\
\hline & & Q & $2.65 \pm 0.02$ & $2.38 \pm 0.01$ & $0.62 \pm 0.01$ & $0.78 \pm 0.04$ \\
\hline & & $\mathrm{W}$ & $2.08 \pm 0.03$ & $1.81 \pm 0.02$ & $0.36 \pm 0.00$ & $0.46 \pm 0.01$ \\
\hline & & $\mathrm{D}$ & $1.29 \pm 0.05$ & $1.24 \pm 0.03$ & $<0.04$ & $>9.41$ \\
\hline \multirow[t]{3}{*}{ 2016-04-24 } & 57503 & $\mathrm{~K}$ & $2.92 \pm 0.07$ & $2.88 \pm 0.05$ & $<1.02$ & $>1.25$ \\
\hline & & Q & $2.99 \pm 0.04$ & $2.62 \pm 0.03$ & $0.39 \pm 0.00$ & $2.19 \pm 0.04$ \\
\hline & & $\mathrm{W}$ & $2.01 \pm 0.04$ & $1.86 \pm 0.03$ & $0.24 \pm 0.03$ & $0.95 \pm 0.25$ \\
\hline \multirow[t]{2}{*}{ 2016-08-23 } & 57624 & $\mathrm{~K}$ & $1.37 \pm 0.02$ & $1.38 \pm 0.02$ & $<1.60$ & $>0.24$ \\
\hline & & $\mathrm{D}$ & $0.87 \pm 0.11$ & $0.76 \pm 0.07$ & $0.24 \pm 0.02$ & $0.19 \pm 0.04$ \\
\hline \multirow[t]{4}{*}{ 2016-10-18 } & 57680 & $\mathrm{~K}$ & $1.19 \pm 0.02$ & $1.19 \pm 0.01$ & $<0.81$ & $>0.82$ \\
\hline & & Q & $1.18 \pm 0.01$ & $1.07 \pm 0.01$ & $0.58 \pm 0.01$ & $0.40 \pm 0.02$ \\
\hline & & $\mathrm{W}$ & $1.02 \pm 0.04$ & $0.86 \pm 0.02$ & $0.32 \pm 0.08$ & $0.28 \pm 0.16$ \\
\hline & & D & $0.87 \pm 0.06$ & $0.66 \pm 0.04$ & $0.36 \pm 0.03$ & $0.08 \pm 0.02$ \\
\hline \multirow[t]{3}{*}{ 2016-11-27 } & 57719 & $\mathrm{~K}$ & $1.49 \pm 0.01$ & $1.49 \pm 0.01$ & $<1.07$ & $>0.58$ \\
\hline & & Q & $1.44 \pm 0.02$ & $1.23 \pm 0.01$ & $0.81 \pm 0.03$ & $0.24 \pm 0.02$ \\
\hline & & $\mathrm{W}$ & $1.31 \pm 0.07$ & $1.12 \pm 0.05$ & $0.36 \pm 0.06$ & $0.28 \pm 0.12$ \\
\hline \multirow[t]{4}{*}{ 2016-12-28 } & 57750 & $\mathrm{~K}$ & $1.95 \pm 0.02$ & $1.95 \pm 0.01$ & $<1.22$ & $>0.59$ \\
\hline & & Q & $1.92 \pm 0.02$ & $1.69 \pm 0.01$ & $0.69 \pm 0.03$ & $0.46 \pm 0.04$ \\
\hline & & $\mathrm{W}$ & $1.50 \pm 0.06$ & $1.23 \pm 0.04$ & $0.40 \pm 0.08$ & $0.26 \pm 0.12$ \\
\hline & & $\mathrm{D}$ & $0.78 \pm 0.04$ & $0.79 \pm 0.03$ & $0.08 \pm 0.01$ & $1.46 \pm 0.61$ \\
\hline
\end{tabular}

Notes. (1) Date in Year-Month-Day, (2) Modified Julian Date, (3) observing frequency band (K, Q, W, and D for 22, 43, 86, and 129 GHz, respectively), (4) observed total flux density in Jy, (5) observed peak flux of core components in Jy, (6) observed core size in mas including its upper limit with a symbol of ' $<$ ', (7) estimated brightness temperature in $10^{10} \mathrm{~K}$ including its lower limit with a symbol of '>'. The uncertainties of 0.00 are less than 0.005 . 